\title{
Arbitrary Source Models and Bayesian Codebooks in Rate-Distortion Theory
}

\author{
Ioannis Kontoyiannis, Member, IEEE, and Junshan Zhang, Member, IEEE
}

\begin{abstract}
We characterize the best achievable performance of lossy compression algorithms operating on arbitrary random sources, and with respect to general distortion measures. Direct and converse coding theorems are given for variable-rate codes operating at a fixed distortion level, emphasizing: a) nonasymptotic results, b) optimal or near-optimal redundancy bounds, and c) results with probability one. This development is based in part on the observation that there is a precise correspondence between compression algorithms and probability measures on the reproduction alphabet. This is analogous to the Kraft inequality in lossless data compression. In the case of stationary ergodic sources our results reduce to the classical coding theorems. As an application of these general results, we examine the performance of codes based on mixture codebooks for discrete memoryless sources. A mixture codebook (or Bayesian codebook) is a random codebook generated from a mixture over some class of reproduction distributions. We demonstrate the existence of universal mixture codebooks, and show that it is possible to universally encode memoryless sources with redundancy of approximately $(d / 2) \log n$ bits, where $d$ is the dimension of the simplex of probability distributions on the reproduction alphabet.
\end{abstract}

Index Terms-Data compression, mixture codebooks, rate-distortion theory, redundancy rate.

\section{INTRODUCTION}

$\mathbf{S}$ UPPOSE data are generated by a random process, or source $\left\{X_{n} ; n \geq 1\right\}$. Roughly speaking, the main objective of data compression is to find efficient representations of data strings $x_{1}^{n}=\left(x_{1}, x_{2}, \ldots, x_{n}\right)$ by variable-length binary strings $\psi_{n}\left(x_{1}^{n}\right)$. If we let $A$ denote the source alphabet, then the map $\psi_{n}: A^{n} \rightarrow\{0,1\}^{*}$ from $A^{n}$ to the set of finite-length binary strings is a (variable-length) block code of length $n$. The compression performance of such a code is described by its length function

$$
L_{n}\left(x_{1}^{n}\right)=\text { length of }\left[\psi_{n}\left(x_{1}^{n}\right)\right] \quad \text { bits, for } x_{1}^{n} \in A^{n} .
$$

In lossless data compression, the natural class of codes to consider is the class of uniquely decodable codes $\psi_{n}$. As is well known, the Kraft inequality (see, e.g., [7, p. 90]) provides a cor-

Manuscript received February 28, 2001; revised March 14, 2002. The work of I. Kontoyiannis was supported in part by the National Science Foundation under Grants 0073378-CCR, DMS-9615444, and by USDA-IFAFS under Grant 00-52100-9615. The work of J. Zhang was supported in part by Arizona State University (ASU) Faculty Initiation Grant DM11001.

I. Kontoyiannis is with the Division of Applied Mathematics and the Department of Computer Science, Brown University, Providence, RI 02912 USA (e-mail: yiannis@dam.brown.edu).

J. Zhang is with the Department of Electrical Engineering, Arizona State University, Tempe, AZ 85287-7206 USA (e-mail: junshan.zhang@asu.edu)

Communicated by P. A. Chou, Associate Editor for Source Coding.

Publisher Item Identifier 10.1109/TIT.2002.800493. respondence between uniquely decodable codes $\psi_{n}$, and probability distributions $Q_{n}$ on $A^{n}$ :

KRAFT INEQUALITY: $(\Leftarrow)$ For any uniquely decodable code $\left(\psi_{n}, L_{n}\right)$ there is a probability measure $Q_{n}$ on $A^{n}$ such that

$$
L_{n}\left(x_{1}^{n}\right) \geq-\log Q_{n}\left(x_{1}^{n}\right) \quad \text { bits, for all } x_{1}^{n} .
$$

$(\Rightarrow)$ Given any probability measure $Q_{n}$ on $A^{n}$ there is a uniquely decodable code $\left(\psi_{n}, L_{n}\right)$ such that

$$
L_{n}\left(x_{1}^{n}\right) \leq-\log Q_{n}\left(x_{1}^{n}\right)+1 \quad \text { bits, for all } x_{1}^{n} .
$$

[Above and throughout the paper, $\log$ denotes the logarithm taken to base 2.] In the first part of the inequality, for $Q_{n}$ we can take the measure

$$
Q_{n}\left(x_{1}^{n}\right) \triangleq Z^{-1} 2^{-L_{n}\left(x_{1}^{n}\right)}
$$

where $Z$ is the normalizing constant

$$
Z=\sum_{x_{1}^{n} \in A^{n}} 2^{-L_{n}\left(x_{1}^{n}\right)} .
$$

Then the usual statement of the Kraft inequality says that $Z \leq 1$.

Turning to lossy compression, we consider the problem of variable-rate coding at a fixed distortion level. More precisely, for each data string $x_{1}^{n}=\left(x_{1}, x_{2}, \ldots, x_{n}\right) \in A^{n}$ produced by the source $\left\{X_{n}\right\}$, our goal is to find an "accurate" representation of $x_{1}^{n}$ by a string $y_{1}^{n}=\left(y_{1}, y_{2}, \ldots, y_{n}\right)$ taking values in the reproduction alphabet $\hat{A}$. The accuracy or "distortion" between two such strings is measured by a family of arbitrary distortion measures $\rho_{n}: A^{n} \times \hat{A}^{n} \rightarrow[0, \infty), n \geq 1$ (more precise definitions will be given later).

The class of codes we consider here is the collection of variable-length codes operating at a fixed distortion level, that is, codes $C_{n}$ defined by triplets $\left(B_{n}, \phi_{n}, \psi_{n}\right)$ where

a) $B_{n}$ is a discrete (finite or countably infinite) subset of $\hat{A}^{n}$, called the codebook;

b) $\phi_{n}: A^{n} \rightarrow B_{n}$ is the encoder or quantizer;

c) $\psi_{n}: B_{n} \rightarrow\{0,1\}^{*}$ is a uniquely decodable representation of the elements of $B_{n}$ by finite-length binary strings.

We say the code $C_{n}=\left(B_{n}, \phi_{n}, \psi_{n}\right)$ operates at distortion level $D$ (for some $D \geq 0$ ), if it encodes each source string with distortion $D$ or less

$$
\rho_{n}\left(x_{1}^{n}, \phi_{n}\left(x_{1}^{n}\right)\right) \leq D, \quad \text { for all } x_{1}^{n} \in A^{n} .
$$


The compression performance of a code $C_{n}$ is described by its length function

$$
\ell_{n}\left(x_{1}^{n}\right)=\text { length of }\left[\psi_{n}\left(\phi_{n}\left(x_{1}^{n}\right)\right)\right] \text { bits. }
$$

For a code $C_{n}$ with associated length function $\ell_{n}$ we will often write $C_{n}=\left(B_{n}, \phi_{n}, \psi_{n}, \ell_{n}\right)$ or simply $\left(C_{n}, \ell_{n}\right)$.

The main theoretical issue of interest here is to characterize the best achievable compression performance of such codes. For stationary ergodic sources, Shannon [25], [36] gave the first such general characterization in terms of the rate-distortion function. In this paper, we adopt a somewhat different point of view. We take, as the starting point, a lossy version of the Kraft inequality, and use that as the basis for the subsequent general development. This approach leads to a natural formulation of the rate-distortion question as a convex selection problem, and allows us to consider, at least for part of the way, completely arbitrary source distributions and distortion measures. This approach has its roots in the earlier work of Bell and Cover [3] and of Kieffer [18].

\section{A. Outline}

Our first main result (part of Theorem 1 in Section II) is the following lossy version of the Kraft inequality. Given a source string $x_{1}^{n} \in A^{n}$ and a distortion level $D \geq 0$, let $B\left(x_{1}^{n}, D\right)$ denote the "distortion-ball" of radius $D$ centered at $x_{1}^{n}$ (see (7) for a precise definition).

LOSSY KRAFT INEQUALITY: $(\Leftarrow)$ For any code

$$
C_{n}=\left(B_{n}, \phi_{n}, \psi_{n}\right)
$$

with associated length function $\ell_{n}$, operating at distortion level $D$, there is a probability measure $Q_{n}$ on $\hat{A}^{n}$ such that

$$
\ell_{n}\left(x_{1}^{n}\right) \geq-\log Q_{n}\left(B\left(x_{1}^{n}, D\right)\right) \text { bits, for all } x_{1}^{n} .
$$

$(\Rightarrow)$ Given any "admissible" sequence of probability measures $\left\{Q_{n}\right\}$ on $\hat{A}^{n}$, there is a sequence of codes

$$
\left\{C_{n}=\left(B_{n}, \phi_{n}, \psi_{n}\right)\right\}
$$

with associated length functions $\left\{\ell_{n}\right\}$, operating at distortion level $D$, such that

$$
\begin{array}{r}
\ell_{n}\left(X_{1}^{n}\right) \leq-\log Q_{n}\left(B\left(X_{1}^{n}, D\right)\right)+(1+\epsilon) \log n \\
\text { bits, eventually, w.p. } 1
\end{array}
$$

where "w.p. 1" above and throughout the paper means "with probability one."

As will become apparent later, the assumption of "admissibility" of the measures $\left\{Q_{n}\right\}$ is simply the natural requirement that random codebooks, generated according to these measures, do not yield codes with infinite rate.

In Theorems 1 and 2 in the following section, it is also shown that the same code performance as in the second part of the lossy Kraft inequality can be achieved in expectation. Further, if for a given sequence of measures $\left\{Q_{n}\right\}$ more detailed information is available on the asymptotic behavior of the "code lengths"

$$
\ell_{n}\left(X_{1}^{n}\right) \approx-\log Q_{n}\left(B\left(X_{1}^{n}, D\right)\right)
$$

then more precise statements can be made about the redundancy achieved by the corresponding codes. The converse part $(\Leftarrow)$ of the lossy Kraft inequality is based on an extension of a simple argument that was implicitly used in [21], and the corresponding direct coding theorems (in contrast to the lossless case) are asymptotic, and they are based on random coding arguments. In order to obtain the precise form of the second-order terms in the description lengths (the terms of order $\log n$ ), extra care is needed in constructing efficient codes.

In view of the codes-measures correspondence implied by the lossy Kraft inequality, the problem of understanding the best achievable compression performance is reduced, at least conceptually, to identifying the "optimal" measures $Q_{n}$ and understanding the exact behavior of the approximate code lengths (1). As we will see, this correspondence leads to a characterization of the achievable performance of compression algorithms not in terms of the rate-distortion function, but in terms of a related quantity $K_{n}(D)$, defined as

$$
K_{n}(D) \triangleq \inf _{Q_{n}} E\left[-\log Q_{n}\left(B\left(X_{1}^{n}, D\right)\right)\right]
$$

where the infimum is over all probability measures $Q_{n}$ on $\hat{A}^{n}$.

Let us assume for a moment that the above infimum is achieved for some $Q_{n}=\tilde{Q}_{n}$. In Theorems 3 and 4, we give both asymptotic and finite- $n$ results on the optimality of the measures $\tilde{Q}_{n}$ and the codes they generate. First, we show that for any code $C_{n}$ with length function $\ell_{n}$ operating at distortion level $D$

$$
E\left[\ell_{n}\left(X_{1}^{n}\right)\right] \geq K_{n}(D) \geq R_{n}(D) \text { bits }
$$

where $R_{n}(D)$ is the $n$ th-order rate distortion function of the source. Then we show that the measures $\tilde{Q}_{n}$ are "competitively optimal" in that, for any measure $Q_{n}$ and any $k>0$

$$
\begin{array}{r}
\operatorname{Pr}\left\{-\log Q_{n}\left(B\left(X_{1}^{n}, D\right)\right) \leq-\log \tilde{Q}_{n}\left(B\left(X_{1}^{n}, D\right)\right)-k\right\} \\
\leq 2^{-k}
\end{array}
$$

(see also Remark 3 after Theorem 3). Moreover, we prove that the codes generated according to the measures $\left\{\tilde{Q}_{n}\right\}$ are asymptotically optimal, up to about $(\log n)$ bits

$$
\begin{array}{r}
-\log Q_{n}\left(B\left(X_{1}^{n}, D\right)\right) \geq-\log \tilde{Q}_{n}\left(B\left(X_{1}^{n}, D\right)\right)-(1+\epsilon) \log n \\
\text { bits, eventually, w.p. 1. }
\end{array}
$$

The statements in (3)-(5) are given in Theorems 3 and 4. Special cases of these results under much more restrictive assumptions (a finite reproduction alphabet $\hat{A}$ and a bounded, single-letter distortion measure) recently appeared in [21].

Note that, so far, no assumptions have been made on the source distribution or the distortion measures $\rho_{n} .{ }^{1}$ As a sanity check, we consider the case of stationary ergodic processes and subadditive distortion measures, and we show in Theorem 5 that, in this case, our general results reduce to Kieffer's pointwise coding theorems in [18], where the quantity $K_{n}(D)$ was defined and used extensively.

\footnotetext{
${ }^{1}$ To be absolutely precise, we should mention that for the results discussed above we do need to make the trivial assumption that finite-rate coding is indeed possible at the distortion level we consider.
} 
As an application of this general framework we consider the problem of universal coding for memoryless sources with respect to single-letter distortion measures. Following the corresponding development in universal lossless compression (see [6] and the references therein), we examine the performance of random codes based on mixture codebooks. Let $\left\{X_{n}\right\}$ be an independent and identically distributed (i.i.d.) source over a finite alphabet $A$, and let the reproduction alphabet $\hat{A}$ also be finite. A mixture codebook (or Bayesian codebook) is a random codebook generated according to sequence of distributions $\left\{M_{n}\right\}$, where each $M_{n}$ is a mixture of i.i.d. distributions $Q^{n}$ on $\hat{A}^{n}$

$$
M_{n}\left(y_{1}^{n}\right) \triangleq \int_{\text {all } Q} Q^{n}\left(y_{1}^{n}\right) d \pi(Q), \quad \text { for } y_{1}^{n} \in \hat{A}^{n} .
$$

In Theorem 6, sufficient conditions are given for the "prior" distribution $\pi$, guaranteeing that the codes generated according to the mixture distributions $\left\{M_{n}\right\}$ are universal over the class of all memoryless sources on $A$.

Under further regularity conditions on the prior $\pi$ (assuming it has a continuous and everywhere positive density with respect to Lebesgue measure), it is shown in Theorem 7 that the redundancy of the mixture codebooks asymptotically does not exceed $\approx(d / 2) \log n$ bits, where $d=|\hat{A}|-1$ is the dimension of the simplex of distributions on the reproduction alphabet $\hat{A}$. Therefore, the price paid for universality is about $(1 / 2) \log n$ bits per degree of freedom, where, in contrast with the case of lossless compression, "freedom" is measured with respect to the class of possible codebook distributions we are allowed to use, and not with respect to the size of the class of sources considered.

In view of the recent results in [28], this rate appears to be optimal and it agrees with the corresponding results in lossless compression [23], [6], as well as with those obtained in [5] within the framework of vector quantization. Moreover, the codes generated by mixture codebooks appear to be the first examples of codes whose redundancy is shown to be near-optimal not only in expectation, but also with probability one.

\section{B. Earlier Work}

A number of relevant papers have already been pointed out and briefly discussed. We also mention that, implicitly, the codes-measures correspondence has been used in the literature by various authors over the past five years or so; relevant works include [22], [27], [29], [19], and [21], among others.

A different approach for dealing with arbitrary sources has been introduced by Steinberg, Verdú, and Han [26], [13], [14], based on the "information-spectrum" method. This leads to a different (asymptotic) characterization of the best achievable performance in lossy data compression. Unlike in those works, more emphasis here has been placed on obtaining nonasymptotic converses, tight redundancy bounds, and coding theorems with probability one rather than in expectation.

The problem of determining the best achievable (expected) redundancy rate in lossy compression was extensively treated in [35]; see also references therein. Suboptimal universal redundancy rates in expectation were computed in [31], [15], and asymptotically tight bounds were recently obtained in [30], [28] where converses were also established. Taking a different point of view, Chou et al. [5] employ high-rate quantization theory to address the question of how close one can come to the optimum performance theoretically achievable (OPTA) function, as opposed to the rate-distortion function. Another related problem, that of characterizing the optimal pointwise redundancy (including the question of universality) has been treated in detail in [21].

All of the works mentioned so far exhibit universal codes based on "multiple codebooks" or "two-stage descriptions." That is, the source string $x_{1}^{n}$ is examined, and based on its statistical properties one of several possible codes is chosen to encode $x_{1}^{n}$. First, the index of the chosen code is communicated to the decoder, then the encoded version of $x_{1}^{n}$ is sent. In contrast, the universal codes presented here are based on a single mixture codebook that works well for all memoryless sources. This construction, facilitated by the codes-measures correspondence, is developed in close analogy to the corresponding lossless compression results; see [8], [23], [6], [2] and references therein. Mixture codebooks for lossy compression are also briefly considered in [34], [33], but with the mixture being over fixed-composition codebooks of a given type, rather than over distributions.

Finally, we note that a different connection between rate-distortion theory and Bayesian inference has been drawn in [32], [16].

\section{STATEMENTS OF RESULTS}

\section{A. The Setting}

We introduce some definitions and notation that will remain in effect throughout the paper. Let $\left\{X_{n} ; n \geq 1\right\}$ be a random process, or source, taking values in the source alphabet $A$, where $A$ is assumed to be a complete, separable metric space, equipped with its Borel $\sigma$-field $\mathcal{A}$. For $1 \leq i \leq \infty$, we write $X_{i}^{j}$ for the vector of random variables $\left(X_{i}, X_{i+1}, \ldots, X_{j}\right)$, and similarly write $x_{i}^{j}=\left(x_{i}, x_{i+1}, \ldots, x_{j}\right) \in A^{j-i+1}$ for a realization of $X_{i}^{j}$. The distribution of $X_{1}^{n}$ is denoted by $P_{n}$ (more precisely, $P_{n}$ is a Borel probability measure on $\left(A^{n}, \mathcal{A}^{n}\right)$ ), and the probability measure describing the distribution of the entire process is denoted by $\mathbb{P}$.

Similarly, for the reproduction alphabet we take $\hat{A}$ to be a complete, separable metric space together with its Borel $\sigma$-field $\hat{\mathcal{A}}$, where $\hat{A}$ may or may not be the same as $A .^{2}$ For each $n \geq 1$, we assume that we are given a distortion measure $\rho_{n}$, that is, a nonnegative function $\rho_{n}: A^{n} \times \hat{A}^{n} \rightarrow[0, \infty){ }^{3}$ For each source string $x_{1}^{n} \in A^{n}$ and distortion level $D \geq 0$ we define the distortion-ball $B\left(x_{1}^{n}, D\right)$ as the collection of all strings $y_{1}^{n} \in \hat{A}^{n}$ that have distortion $D$ or less with respect to $x_{1}^{n}$

$$
B\left(x_{1}^{n}, D\right) \triangleq\left\{y_{1}^{n} \in \hat{A}^{n}: \rho_{n}\left(x_{1}^{n}, y_{1}^{n}\right) \leq D\right\} .
$$

Finally, throughout the paper, $\log$ denotes the logarithm taken to base 2 and $\log _{e}$ denotes the natural logarithm. Unless explicitly stated otherwise, all familiar information-theoretic quantities (the relative entropy, rate-distortion function, and so on) are

\footnotetext{
${ }^{2}$ To avoid uninteresting technicalities, we assume throughout that all singletons are measurable, i.e., $\{x\} \in \mathcal{A}$ and $\{y\} \in \hat{\mathcal{A}}$ for all $x \in A, y \in \hat{A}$.

${ }^{3}$ Assuming, of course, that each $\rho_{n}$ is measurable with respect to the product $\sigma$-field $\mathcal{A}^{n} \times \hat{\mathcal{A}}^{n}$.
} 
defined in terms of logarithms taken to base 2 , and are hence expressed in bits.

\section{B. Random Codebooks}

Given an arbitrary (Borel) probability measure $Q_{n}$ on $\hat{A}^{n}$, by a random codebook generated according to $Q_{n}$ we mean a collection of independent random vectors

$$
Y(i)=Y_{1}^{n}(i), \quad i \geq 1
$$

taking values in $\hat{A}^{n}$, each generated according to the measure $Q_{n}$. These random vectors will serve as the codebook in various direct coding theorems presented later. Given a random source string $X_{1}^{n}$, a random codebook as above, and a distortion level $D \geq 0$, we define the waiting time $W_{n}$ as the index $i$ of the first element of the codebook that matches $X_{1}^{n}$ with distortion $D$ or less

$$
W_{n} \triangleq \inf \left\{i \geq 1: \rho_{n}\left(X_{1}^{n}, Y_{1}^{n}(i)\right) \leq D\right\}
$$

with the usual convention that the infimum of the empty set equals $\infty$.

In the coding scenario, we assume that the random codebook is available to both the encoder and the decoder. The gist of the subsequent direct coding theorems is to find efficient ways for the encoder to communicate to the decoder the value of the waiting time $W_{n}$; once this is available, the decoder can read off the codeword $Y_{1}^{n}\left(W_{n}\right)$ and obtain a $D$-close version of $X_{1}^{n}$.

It is perhaps worth mentioning that the relationship of the $n$th codebook to the $(n+1)$ st codebook will be irrelevant in all our subsequent direct coding arguments. For the sake of being specific it may be convenient to think of codebooks with different block lengths as being independent. On the other hand, if $Q_{n}$ and $Q_{n+1}$ are consistent measures, i.e., if $Q_{n}$ happens to be the $n$-dimensional measure induced by $Q_{n+1}$ on $\hat{A}^{n}$, then the $(n+1)$ st codebook can be generated from the $n$th one by extending each of its codewords by an additional letter generated according to the conditional distribution induced by $Q_{n+1}$.

\section{Arbitrary Sources}

Let $\left\{X_{n}\right\}$ be an arbitrary source and $\left\{\rho_{n}\right\}$ a given sequence of distortion measures as above. At various points below we will need to impose the following assumptions. They are variations of $\left[18\right.$, Condition $\left.\left.2^{\prime}\right)\right]$.

(WQC): For a distortion level $D \geq 0$ we say that the weak quantization condition (WQC) holds at level $D$ if for each $n$ there is a (measurable) quantizer $q^{(n)}: A^{n} \rightarrow B_{n} \subset \hat{A}^{n}$ such that $B_{n}$ is a finite or countably infinite set, and

$$
\rho_{n}\left(x_{1}^{n}, q^{(n)}\left(x_{1}^{n}\right)\right) \leq D, \quad \text { for all } x_{1}^{n} \in A^{n} .
$$

(QC): For a distortion level $D \geq 0$ we say that the quantization condition (QC) holds at level $D$, if (WQC) holds with respect to quantizers $q^{(n)}$ of finite rate

$$
M_{1} \triangleq \sup _{n \geq 1} \frac{1}{n} H\left(q^{(n)}\left(X_{1}^{n}\right)\right)<\infty
$$

where $H\left(q^{(n)}\left(X_{1}^{n}\right)\right)$ denotes the entropy (in bits) of the discrete random variable $q^{(n)}\left(X_{1}^{n}\right)$.
( $p \mathrm{SQC})$ : For a distortion level $D \geq 0$ we say that the $p$-strong quantization condition ( $p S Q C$ ) holds at level $D$ for some $1 \leq$ $p \leq \infty$, if (WQC) holds with respect to quantizers $q^{(n)}$ also satisfying

$$
M_{p} \triangleq \sup _{n \geq 1} \frac{1}{n}\left\{E\left[\left(-\log \mu_{n}\left(q^{(n)}\left(X_{1}^{n}\right)\right)\right)^{p}\right]\right\}^{1 / p}<\infty
$$

where $\mu_{n}$ denotes the (discrete) distribution of $q^{(n)}\left(X_{1}^{n}\right)$ on $\hat{A}^{n}$. [For $p=\infty$ the above expression is interpreted, as usual, to be the corresponding $L_{\infty}$ norm, i.e., $M_{\infty}=\sup _{n}(1 / n)$ $\left.\cdot\left\|-\log \mu_{n}\left(q^{(n)}\left(X_{1}^{n}\right)\right)\right\|_{\infty} \cdot\right]$

Note that clearly $(p S Q C) \Rightarrow(Q C) \equiv(1 \mathrm{SQC}) \Rightarrow(\mathrm{WQC})$. Also observe that, if $\left\{X_{n}\right\}$ is a stationary source and the $\left\{\rho_{n}\right\}$ are single-letter (or subadditive) distortion measures, then each of the above three conditions reduces to the existence of a suitable scalar quantizer, that is, each quantization condition reduces to the corresponding requirement for $n=1$.

For the statement of the next theorem we also need the following definitions. For each $n \geq 1$, let $Q_{n}$ be a probability measure on $\hat{A}^{n}$. The sequence $\left\{Q_{n}\right\}$ is called admissible if the random codebooks generated according to these measures yield codes with finite rate, with probability one. Formally, $\left\{Q_{n}\right\}$ is admissible if there is a finite constant $R$ such that

$$
\limsup _{n \rightarrow \infty}-\frac{1}{n} \log Q_{n}\left(B\left(X_{1}^{n}, D\right)\right) \leq R<\infty \quad \text { w.p. } 1 .
$$

Similarly, the sequence $\left\{Q_{n}\right\}$ is called admissible in expectation if it yields random codes with finite average rate, that is, ${ }^{4}$

$$
R=\limsup _{n \rightarrow \infty} \frac{1}{n} E\left[-\log Q_{n}\left(B\left(X_{1}^{n}, D\right)\right)\right]<\infty .
$$

The following result demonstrates the correspondence between sequences of codes $C_{n}$ operating at distortion level $D$ and sequences of measures $Q_{n}$ on the reproduction spaces $\hat{A}^{n}$. The theorem is proved in Section III-A.

Theorem 1. Codes-Measures Correspondence: Given a distortion level $D \geq 0$, assume that condition (WQC) holds at level $D$.

i) For any code

$$
C_{n}=\left(B_{n}, \phi_{n}, \psi_{n}, \ell_{n}\right)
$$

operating at distortion level $D$ there is a probability measure $Q_{n}$ on $\hat{A}^{n}$ such that

$$
\ell_{n}\left(x_{1}^{n}\right) \geq-\log Q_{n}\left(B\left(x_{1}^{n}, D\right)\right) \text { bits, for all } x_{1}^{n} \in A^{n} .
$$

ii) For any admissible sequence of probability measures $\left\{Q_{n}\right\}$ there is a sequence of codes

$$
\left\{C_{n}=\left(B_{n}, \phi_{n}, \psi_{n}, \ell_{n}\right)\right\}
$$

operating at distortion level $D$ such that

$$
\begin{array}{r}
\ell_{n}\left(X_{1}^{n}\right) \leq-\log Q_{n}\left(B\left(X_{1}^{n}, D\right)\right)+\log n+3 \log \log n \\
+ \text { Const. bits, eventually, w.p. } 1 .
\end{array}
$$

${ }^{4}$ Note that, for any probability measure $Q_{n}$ on $\left(\hat{A}^{n}, \hat{\mathcal{A}}^{n}\right)$, the function $x_{1}^{n} \mapsto$ $-\log Q_{n}\left(B\left(x_{1}^{n}, D\right)\right)$ is measurable with respect to $\mathcal{A}^{n}$. To see this, simply observe that we can take $Q_{n}\left(B\left(x_{1}^{n}, D\right)\right)$ to be the regular conditional probability of the event $\left\{\rho_{n}\left(X_{1}^{n}, Y_{1}^{n}\right) \leq D\right\}$ with respect to $P_{n} \times Q_{n}$, conditional on the $\sigma$-field $\mathcal{A}^{n}$. 
iii) For any sequence of probability measures $\left\{Q_{n}\right\}$ that are admissible in expectation, there is a sequence of codes

$$
\left\{C_{n}=\left(B_{n}, \phi_{n}, \psi_{n}, \ell_{n}\right)\right\}
$$

operating at distortion level $D$ such that

$$
\begin{aligned}
E\left[\ell_{n}\left(X_{1}^{n}\right)\right] \leq & E\left[-\log Q_{n}\left(B\left(X_{1}^{n}, D\right)\right)\right]+\log n \\
& +2 \log \log n+\text { Const. bits, eventually. }
\end{aligned}
$$

Remark 1: The constant terms in parts ii) and iii) of Theorem 1 depend only on the constant $R$ that bounds the asymptotic rate of the measures $Q_{n}$ in (9) and (10), respectively.

Our next result shows that the redundancy rates of the codes in parts ii) and iii) of Theorem 1 can be improved when we have more information about the asymptotic behavior of the approximate code lengths (1) corresponding to the measures $Q_{n}$. Theorem 2 is proved in Section III-B.

\section{Theorem 2. Improved Redundancy Rates:}

i) Given a distortion level $D \geq 0$, assume that condition (WQC) holds at level $D$. Let $\left\{Q_{n}\right\}$ be an admissible sequence of probability measures and assume that

$$
\begin{aligned}
Z_{n} & \triangleq\left|-\log Q_{n}\left(B\left(X_{1}^{n}, D\right)\right)-n R\right| \\
& \leq B \sqrt{n} \log n \quad \text { eventually, w.p. } 1
\end{aligned}
$$

for some finite constant $B$. Then there are codes

$$
\left\{C_{n}=\left(B_{n}, \phi_{n}, \psi_{n}, \ell_{n}\right)\right\}
$$

operating at distortion level $D$ such that

$$
\begin{aligned}
\ell_{n}\left(X_{1}^{n}\right) \leq & -\log Q_{n}\left(B\left(X_{1}^{n}, D\right)\right)+\frac{1}{2} \log n \\
& +2 \log \log n+\text { Const. bits, eventually, w.p. } 1 .
\end{aligned}
$$

ii) Given a distortion level $D \geq 0$, assume that condition ( $p \mathrm{SQC}$ ) holds at level $D$ for some $p \geq 2$. Assume that the sequence $\left\{Q_{n}\right\}$ is admissible in expectation, and let $Z_{n}$ be defined as above. If for $n$ large enough and for some finite nonzero constants $B$ and $C$

$$
\operatorname{Pr}\left\{Z_{n}>B \sqrt{n} \log n\right\} \leq \frac{C}{n^{q}}
$$

where $1 / p+1 / q=1$, then there are codes

$$
\left\{C_{n}=\left(B_{n}, \phi_{n}, \psi_{n}, \ell_{n}\right)\right\}
$$

operating at distortion level $D$ such that

$$
\begin{aligned}
E\left[\ln _{n}\left(X_{1}^{n}\right)\right] \leq E[ & \left.-\log Q_{n}\left(B\left(X_{1}^{n}, D\right)\right)\right]+\frac{1}{2} \log n \\
& +2 \log \log n+\text { Const. bits, eventually. }
\end{aligned}
$$

Remark 2: The constant term in the statement of part i) of Theorem 2 depends only on the constant $B$ in (11). The constant term in part ii) of Theorem 2 depends only on the constants $B$, $C$, and the constant $M_{p}$ in the ( $p \mathrm{SQC}$ ) condition (8).

Note that in many important cases of interest, the assumptions of Theorems 1 and 2 on the asymptotic behavior of the approximate code lengths (1) can be verified via the "generalized asymptotic equipartition property (AEP)" and its refinements; see [10] for an extensive discussion.
Theorems 1 and 2 indicate that the best achievable performance of codes operating at distortion level $D$ can be understood almost entirely on the basis of understanding the precise behavior of the approximate code lengths (1). To this end, for each $n \geq 1$ and distortion level $D \geq 0$ we define (cf. (2)) the quantity

$$
K_{n}(D) \triangleq \inf _{Q_{n}} E\left[-\log Q_{n}\left(B\left(X_{1}^{n}, D\right)\right)\right]
$$

where the infimum is over all probability measures $Q_{n}$ on $\hat{A}^{n}$.

The next theorem gives finite- $n$ bounds on the achievable compression performance of arbitrary codes operating at distortion level $D$. In particular, it identifies the measures $Q_{n}=\tilde{Q}_{n}$ that are optimal in terms of compression performance, as those that achieve the infimum in the definition of $K_{n}(D)$.

Theorem 3. Nonasymptotic Bounds:

i) For any code $\left(C_{n}, \ell_{n}\right)$ operating at distortion level $D$

$$
E\left[\ell_{n}\left(X_{1}^{n}\right)\right] \geq K_{n}(D) \geq R_{n}(D)
$$

where $R_{n}(D)$ is the usual $n$ th-order rate-distortion function of the source $\left\{X_{n}\right\}$ [4], defined by

$$
R_{n}(D)=\inf _{\left(X_{1}^{n}, Y_{1}^{n}\right): X_{1}^{n} \sim P_{n} \text { and } E\left[\rho_{n}\left(X_{1}^{n}, Y_{1}^{n}\right)\right] \leq D} I\left(X_{1}^{n} ; Y_{1}^{n}\right)
$$

where $I\left(X_{1}^{n} ; Y_{1}^{n}\right)$ denotes the mutual information between $X_{1}^{n}$ and $Y_{1}^{n}$, and the infimum is taken over all jointly distributed random vectors $\left(X_{1}^{n}, Y_{1}^{n}\right)$ with $X_{1}^{n}$ having the source distribution $P_{n}$, and

$$
E\left[\rho_{n}\left(X_{1}^{n}, Y_{1}^{n}\right)\right] \leq D .
$$

ii) Assume that the infimum in the definition of $K_{n}(D)$ is achieved by some probability measure $\tilde{Q}_{n}$ and that $K_{n}(D)<\infty$. Then for any probability measure $Q_{n}$ on $\hat{A}^{n}$ we have

$$
E\left[\frac{Q_{n}\left(B\left(X_{1}^{n}, D\right)\right)}{\tilde{Q}_{n}\left(B\left(X_{1}^{n}, D\right)\right)}\right] \leq 1
$$

and for any $k>0$

$$
\begin{array}{r}
\operatorname{Pr}\left\{-\log Q_{n}\left(B\left(X_{1}^{n}, D\right)\right) \leq-\log \tilde{Q}_{n}\left(B\left(X_{1}^{n}, D\right)\right)-k\right\} \\
\leq 2^{-k}
\end{array}
$$

Remark 3. Competitive Optimality: The second result in part ii) of Theorem 3 is somewhat striking. It states that, for any fixed $n$, there is an optimal code operating at distortion level $D$ (the code corresponding to $\tilde{Q}_{n}$ ) with the following property. The probability that any other code beats the optimal one by $k$ or more bits is at most $2^{-k}$. For a detailed example see [20, Sec. IV].

Remark 4. Achievability: Although in general there may not exist measures $\tilde{Q}_{n}$ achieving the infimum in the definition of $K_{n}(D)$, when $\hat{A}$ is finite and $\left\{\rho_{n}\right\}$ is a sequence of bounded, single-letter distortion measures such $\tilde{Q}_{n}$ were shown to exist in [21]. When the infimum is not achieved (or the achievability is not easy to check), it is still possible to recover the result of part ii) of Theorem 3; see Remark 7 after Theorem 4.

Remark 5. $K_{n}(D)$ Versus $R_{n}(D)$ : The function $K_{n}(D)$ can be defined in more familiar information-theoretic terms, 
making it more easily comparable to the rate-distortion function $R_{n}(D)$-see Lemma 1 later. In that form, $K_{n}(D)$ is reminiscent of Kolmogorov's definition of $\epsilon$-entropy (which explains the choice of the letter $K$ in the notation). It is obvious from Lemma 1 that $K_{n}(D)$ is generally larger than $R_{n}(D)$, but for stationary-ergodic sources their limiting values

$$
\lim _{n}(1 / n) K_{n}(D) \text { and } \lim _{n}(1 / n) R_{n}(D)
$$

are equal (see Theorem 5). Lemma 1 is proved in Appendix I.

Lemma 1. Alternative Characterization of $K_{n}(D)$ : For all $D \geq 0$ we have

$$
K_{n}(D)=\underset{\left(X_{1}^{n}, Y_{1}^{n}\right): \rho_{n}\left(X_{1}^{n}, Y_{1}^{n}\right) \leq D \text { w.p.1 }}{ } I\left(X_{1}^{n} ; Y_{1}^{n}\right)
$$

where the infimum is taken over all jointly distributed random vectors $\left(X_{1}^{n}, Y_{1}^{n}\right)$ with $X_{1}^{n}$ having the source distribution $P_{n}$, and $\rho_{n}\left(X_{1}^{n}, Y_{1}^{n}\right) \leq D$ with probability one.

Next we deduce the following asymptotic result from Theorem 3: Up to approximately $\log n$ bits, the code lengths

$$
\ell_{n}\left(X_{1}^{n}\right) \approx-\log \tilde{Q}_{n}\left(B\left(X_{1}^{n}, D\right)\right)
$$

are both achievable and impossible to beat, with probability one. Since the proofs of Theorems 3 and 4 follow very closely along the lines of the corresponding results in [21] we only include brief outlines of their proofs in Section III-C.

Theorem 4. Asymptotic Bounds: Assume that for all $n$ large enough, the infimum in the definition of $K_{n}(D)$ is achieved by some probability measure $\tilde{Q}_{n}$, and that $K_{n}(D)<\infty$.

i) For any sequence of codes $\left\{C_{n}, \ell_{n}\right\}$ operating at distortion level $D$

$$
\begin{array}{r}
\ell_{n}\left(X_{1}^{n}\right) \geq-\log \tilde{Q}_{n}\left(B\left(X_{1}^{n}, D\right)\right)-\log n-2 \log \log n \\
\text { bits, eventually, w.p. } 1 .
\end{array}
$$

ii) Moreover, if the sequence $\left\{\tilde{Q}_{n}\right\}$ is admissible and condition (WQC) holds at level $D$, then there is a sequence of codes $\left\{C_{n}, \ell_{n}\right\}$ operating at distortion level $D$ such that

$$
\begin{array}{r}
\ell_{n}\left(X_{1}^{n}\right) \leq-\log \tilde{Q}_{n}\left(B\left(X_{1}^{n}, D\right)\right)+\log n+3 \log \log n \\
+ \text { Const. bits, eventually, w.p. } 1 .
\end{array}
$$

Similarly, if the sequence $\left\{\tilde{Q}_{n}\right\}$ is admissible in expectation then there exist codes so that (12) holds in expectation, and with $2 \log \log n$ in place of $3 \log \log n$.

Remark 6. Admissibility: The assumption that the optimal measures $\left\{\tilde{Q}_{n}\right\}$ are admissible is typically not restrictive. For example, as we will see in the next section, they are always admissible (as well as admissible in expectation) when the source is stationary and ergodic.

Remark 7. More on Achievability: If $K_{n}(D)<\infty$ but the infimum in the definition of $K_{n}(D)$ is not achieved, there always exists a function $\tilde{g}_{n}: A^{n} \rightarrow[0, \infty)$ in the $L^{1}$-closure of the collection $\mathcal{G}_{n}$ of functions on $A^{n}$ defined by

$$
\begin{array}{r}
\mathcal{G}_{n} \triangleq\left\{g \in L^{1}\left(P_{n}\right): g\left(x_{1}^{n}\right) \geq-\log Q_{n}\left(B\left(x_{1}^{n}, D\right)\right)\right. \\
\left.\quad \text { for a probability measure } Q_{n} \text { on } \hat{A}^{n}\right\}
\end{array}
$$

such that $K_{n}(D)=E\left[\tilde{g}_{n}\left(X_{1}^{n}\right)\right]$; see [17, Theorem 1]. Moreover, the conclusions of part ii) of Theorem 3 and the converse in part i) of Theorem 4 all still hold if we replace the term $-\log \tilde{Q}_{n}\left(B\left(X_{1}^{n}, D\right)\right)$ by $\tilde{g}_{n}\left(X_{1}^{n}\right)$.

\section{Ergodic Sources}

We briefly consider the case of stationary ergodic sources and demonstrate how the classical coding theorems follow from the general results above. Assume that the source $\left\{X_{n}\right\}$ is stationary and ergodic, and that the distortion measures $\left\{\rho_{n}\right\}$ are subadditive, i.e.,

$$
\begin{aligned}
(m+n) \rho_{m+n}\left(x_{1}^{m+n}, y_{1}^{m+n}\right) \leq m \rho_{m}( & \left.x_{1}^{m}, y_{1}^{m}\right) \\
& +n \rho_{n}\left(x_{m+1}^{m+n}, y_{m+1}^{m+n}\right)
\end{aligned}
$$

for all $x_{1}^{m+n} \in A^{m+n}, y_{1}^{m+n} \in \hat{A}^{m+n}$, and all $m, n \geq 1$. We will also assume the existence of a reference letter $\hat{a} \in \hat{A}$ such that

$$
E\left[\rho_{1}\left(X_{1}, \hat{a}\right)\right]<\infty .
$$

The following theorem is essentially due to Kieffer [18]. To connect it with our earlier results we include a brief outline of its proof in Appendix II.

Theorem 5. Stationary-Ergodic Sources [18]: Let $\left\{X_{n}\right\}$ be a stationary-ergodic source such that condition (QC) holds for all $D>0$. Assume that the distortion measures $\left\{\rho_{n}\right\}$ are subadditive, and that a reference letter exists. Then $K_{n}(D)$ is finite for all $n \geq 1$, and the limit

$$
K(D) \triangleq \lim _{n \rightarrow \infty} \frac{1}{n} K_{n}(D)
$$

exists and is equal to the rate-distortion function of the source $\left\{X_{n}\right\}$

$$
R(D) \triangleq \lim _{n \rightarrow \infty} \frac{1}{n} R_{n}(D) .
$$

If, moreover, for all $n$ large enough the infimum in the definition of $K_{n}(D)$ is achieved by some probability measure $\tilde{Q}_{n}$, then we also have

i) As $n \rightarrow \infty$

$$
\begin{aligned}
&-\frac{1}{n} \log \tilde{Q}_{n}\left(B\left(X_{1}^{n}, D\right)\right) \rightarrow K(D)=R(D) \\
& \text { w.p. } 1 \text { and in } L^{1} .
\end{aligned}
$$

ii) For any sequence of codes $\left\{C_{n}, \ell_{n}\right\}$ operating at distortion level $D$

$$
\liminf _{n \rightarrow \infty} \frac{1}{n} \ell_{n}\left(X_{1}^{n}\right) \geq K(D)=R(D) \quad \text { bits/symbol, w.p. } 1
$$

and there exist codes achieving this bound with equality.

Remark 8. Even More on Achievability: As described in Remark 7, even when the achieving measure $\tilde{Q}_{n}$ does not exist we can always find a function $\tilde{g}_{n} \in L^{1}\left(P_{n}\right)$ so that $\tilde{g}_{n}\left(X_{1}^{n}\right)$ plays the role of $-\log \tilde{Q}_{n}\left(B\left(X_{1}^{n}, D\right)\right)$. In this context, [17, Theorem 2] implies that part i) of Theorem 5 always holds with $\tilde{g}_{n}\left(X_{1}^{n}\right)$ in place of $-\log \tilde{Q}_{n}\left(B\left(X_{1}^{n}, D\right)\right)$, and, therefore, the pointwise converse (15) is also always valid.

\section{E. Mixture Codebooks and Memoryless Sources}

As we saw in Theorems 3 and 4, the optimal measures $\widetilde{Q}_{n}$ completely characterize the best compression performance of 
codes operating at a fixed distortion level. But the $\tilde{Q}_{n}$ themselves are typically hard to describe explicitly.

In this subsection, we restrict our attention to memoryless sources. Although it is not hard to see that even here the measures $\tilde{Q}_{n}$ do not have a particularly simple structure, we do know [35], [29], [21] that, when dealing with a single memoryless source, asymptotically optimal compression can still be achieved by codes based on product measures, i.e., measures $Q_{n}$ of the form $Q_{n}=Q^{n}$ on $\hat{A}^{n}$. Taking $Q^{*}$ to be the optimal reproduction distribution at distortion level $D$, the codes generated according to the product measures $\left(Q^{*}\right)^{n}$ achieve near-optimal redundancy both in expectation and with probability one (see Proposition 1 and the discussion following Theorem 7 for details).

Turning to the problem of universal coding, the above discussion motivates us to consider codes based on random codebooks that are generated according to mixtures over the class of all product distributions $Q^{n}$ on $\hat{A}^{n}$. The existence of universal mixture codebooks will be established, and sufficient conditions will be given under which the redundancy rate they achieve is optimal.

For the remainder of this section, $\left\{X_{n}\right\}$ is assumed to be a stationary memoryless source, that is, the random variables $X_{n}$ are i.i.d, with common distribution $P$ on $A$. We take both $A$ and $\hat{A}$ to be finite sets and write $|\hat{A}|=d+1$ for the cardinality of $\hat{A}$. Given a distortion measure $\rho: A \times \hat{A} \rightarrow[0, \infty)$, we consider the family of single-letter distortion measures $\left\{\rho_{n}\right\}$

$\rho_{n}\left(x_{1}^{n}, y_{1}^{n}\right) \triangleq \frac{1}{n} \sum_{i=1}^{n} \rho\left(x_{i}, y_{i}\right), \quad x_{1}^{n} \in A^{n}, y_{1}^{n} \in \hat{A}^{n}, n \geq 1$.

We also make the customary assumption that for each $a \in A$ there is an $\hat{a} \in \hat{A}$ such that $\rho(a, \hat{a})=0$.

Following [27], [21], for each probability distribution $Q$ on $\hat{A}$ and all $D \geq 0$, we define the rate function

$R(P, Q, D)$

$$
\triangleq \inf _{(X, Y): X \sim P, E[\rho(X, Y)] \leq D}\left\{I(X ; Y)+H\left(Q_{Y} \| Q\right)\right\}
$$

where $H(\cdot \| \cdot)$ denotes the relative entropy, the infimum is taken over all jointly distributed random variables $(X, Y)$ such that $X$ has the source distribution $P$ and $E[\rho(X, Y)] \leq D$, and $Q_{Y}$ denotes the marginal distribution of $Y$. It is not hard to see that $R(P, Q, D)$ is related to the rate-distortion function $R(D)$ of the source $P$ via

$$
R(D)=\inf _{Q} R(P, Q, D)=R\left(P, Q^{*}, D\right)
$$

where the infimum is taken over all probability distributions $Q$ on $\hat{A}$; cf. [27], [21]. We let $Q_{n}^{*}$ denote the product measures $\left(Q^{*}\right)^{n}$ on $\hat{A}^{n}, n \geq 1$, and we call the measures $Q_{n}^{*}$ the optimal reproduction distributions at distortion level $D$ (even though the achieving $Q^{*}$ in (16) may not be unique).

The following proposition shows that $R(P, Q, D)$ characterizes the first-order compression performance of the random codebooks generated according to the product measures $Q^{n}$. In particular (recall Theorem 1), it implies that the codebooks generated according to the optimal reproduction distributions $\left\{Q_{n}^{*}\right\}$ achieve first-order optimal compression performance.
Proposition 1 [27]: For any probability measure $Q$ on $\hat{A}$, and for all $D>0$

$$
\lim _{n \rightarrow \infty}-\frac{1}{n} \log Q^{n}\left(B\left(X_{1}^{n}, D\right)\right)=R(P, Q, D) \quad \text { w.p. } 1 .
$$

Next define as usual

$$
D_{\max }=D_{\max }(P)=\min _{y \in \hat{A}} E_{P}[\rho(X, y)] .
$$

Since $R(D)=0$ for $D \geq D_{\max }$, to avoid the trivial case when $R(D)$ is identically equal to zero we assume that $D_{\max }>0$. Also, from now on, we restrict our attention to distortion levels $D$ in the interesting range $D \in\left(0, D_{\max }\right)$.

Let $\mathcal{P}$ denote the $d$-dimensional simplex of probability measures $Q$ on $\hat{A}$, and let $\pi$ be a probability measure on $\mathcal{P}$; we refer to $\pi$ as the "prior" distribution on $\mathcal{P}$. For each $n \geq 1$, we define the mixture distribution $M_{n}$ on $\hat{A}^{n}$ as in (6)

$$
M_{n}\left(y_{1}^{n}\right)=\int_{Q \in \mathcal{P}} Q^{n}\left(y_{1}^{n}\right) d \pi(Q), \quad \text { for } y_{1}^{n} \in \hat{A}^{n} .
$$

The next theorem gives a simple sufficient condition on the prior, under which the first-order performance of codes based on the mixture distributions $M_{n}$ is universally optimal. Theorem 6 is proved in Section IV-A using an argument similar to that used in the proof of the corresponding lossless result in [1].

Theorem 6. Universality of i.i.d. Mixtures: Let $\left\{X_{n}\right\}$ be an i.i.d. source with distribution $P$ on $A$. For $D \in\left(0, D_{\max }\right)$, let $Q^{*}$ denote the optimal reproduction distribution of $P$ at distortion level $D$. If the prior $\pi$ has a density $p$ with respect to Lebesgue measure on the simplex, and $p$ is strictly positive in a neighborhood of $Q^{*}$, then

$$
\begin{aligned}
-\log M_{n}\left(B\left(X_{1}^{n}, D\right)\right) \leq & -\log Q_{n}^{*}\left(B\left(X_{1}^{n}, D\right)\right) \\
& +o(n) \quad \text { w.p. } 1, \text { as } n \rightarrow \infty .
\end{aligned}
$$

More generally, (19) remains valid as long as the prior $\pi$ assigns positive mass to all neighborhoods

$$
\begin{array}{r}
N\left(Q^{*}, \epsilon\right) \triangleq\left\{Q: R(P, Q, D)<R\left(P, Q^{*}, D\right)+\epsilon\right\}, \\
\text { for } \epsilon>0 .
\end{array}
$$

The conditions of Theorem 6 are easily seen to be satisfied, e.g., when the prior $\pi$ has an everywhere strictly positive density $p$, or when $\pi$ assigns positive mass to the optimal reproduction distribution itself. In particular, this includes the special case of discrete mixtures of the form

$$
M_{n}=\sum_{i=1}^{\infty} w_{i} Q_{i}^{n}
$$

where $Q^{*}$ is one of the mixing distributions $Q_{i}$. More generally, the discrete mixtures $M_{n}$ are universal if the $\left\{Q_{i}\right\}$ are a countable dense subset of the simplex $\mathcal{P}$ and $\left\{w_{i}\right\}$ are strictly positive weights summing to one.

Remark 9. Universal Codes: Suppose that the assumptions of Theorem 6 hold for all $Q^{*}$ on the simplex-e.g., take $\pi$ to be the normalized Lebesgue measure on the simplex or a discrete probability measure supported on a countable dense set. Then, 
in view of Proposition 1 and (16), Theorem 6 implies that the mixtures measures $\left\{M_{n}\right\}$ have

$$
\limsup _{n \rightarrow \infty}-\frac{1}{n} \log M_{n}\left(B\left(X_{1}^{n}, D\right)\right) \leq R(D) \quad \text { w.p. } 1 .
$$

Therefore they are admissible, and Theorem 1 implies the existence of universal codes over the class of all memoryless sources on $A$.

Next it is shown that when the prior $\pi$ satisfies certain smoothness conditions, the asymptotic redundancy rate of the mixture codebooks is approximately $(d / 2) \log n$ bits, where $d=|\hat{A}|-1$ is the dimensionality of $\mathcal{P}$.

For Theorem 7, we only consider sources $P$ that have $P(a)>$ 0 for all $a \in A$, and we assume that the optimal reproduction distribution $Q^{*}$ is unique and is achieved in the interior of the simplex. Formally, for each $D \geq 0$ we consider sources $P$ in the collection

$$
\begin{aligned}
\mathcal{S}(D) \triangleq\{ & P: D<D_{\max }(P), Q^{*} \text { is unique, } \\
& \left.\quad \operatorname{support}(P)=A, \text { and } \operatorname{support}\left(Q^{*}\right)=\hat{A}\right\} .
\end{aligned}
$$

Theorem 7. Pointwise Redundancy of i.i.d. Mixtures: Let $D>0$, and assume that the prior $\pi$ has a strictly positive, continuous density $p$ with respect to Lebesgue measure. Then for any source distribution $P$ in $\mathcal{S}(D)$

$$
\begin{array}{r}
-\log M_{n}\left(B\left(X_{1}^{n}, D\right)\right) \leq-\log Q_{n}^{*}\left(B\left(X_{1}^{n}, D\right)\right)+\frac{d}{2} \log n \\
+O(\log \log n) \quad \text { w.p. } 1 \text {, as } n \rightarrow \infty
\end{array}
$$

where $Q_{n}^{*}$ are the optimal reproduction distributions for the source $P$ at distortion level $D$.

The proof of Theorem 7 (given in Section IV-C) is based on an argument using Laplace's method of integration, and closely parallels the proof of the corresponding result of Clarke and Barron [6] in the lossless case. Using (22) and applying Theorem 2, we get a sequence of universal codes with near-optimal redundancy rate.

Corollary 1. Universal Pointwise Redundancy: Let $D>0$. There is a sequence of codes

$$
\left\{C_{n}=\left(B_{n}, \phi_{n}, \psi_{n}, \ell_{n}\right)\right\}
$$

operating at distortion level $D$ such that, for all memoryless sources $P$ in $\mathcal{S}(D)$

$$
\begin{aligned}
\ell_{n}\left(X_{1}^{n}\right) \leq-\log Q_{n}^{*}\left(B\left(X_{1}^{n}, D\right)\right)+\left(\frac{d+1}{2}\right) \log n \\
+O(\log \log n) \quad \text { bits, w.p. } 1, \text { as } n \rightarrow \infty
\end{aligned}
$$

where $Q_{n}^{*}$ are the optimal reproduction distributions for the source $P$ at distortion level $D$.

Corollary 1 follows from combining Theorem 7 with part i) of Theorem 2. To verify that assumptions of part i) of Theorem 2 are satisfied, recall [21, Proposition 3].

\section{Remarks:}

a) Interpretation: What Theorem 7 and Corollary 1 really tell us is that the codes corresponding to the mixture distri- butions $\left\{M_{n}\right\}$ have code lengths that do not exceed the "optimum"

$$
\ell_{n}^{*}\left(X_{1}^{n}\right) \triangleq-\log Q_{n}^{*}\left(B\left(X_{1}^{n}, D\right)\right)+\frac{1}{2} \log n \quad \text { bits }
$$

by more than $\approx(d / 2) \log n$ bits. Why call the code lengths $\ell_{n}^{*}\left(X_{1}^{n}\right)$ optimum? In view of Theorem 2, $\ell_{n}^{*}\left(X_{1}^{n}\right)$ correspond to random codes generated according to the measures $\left\{Q_{n}^{*}\right\}$. In [29], [35], and in [21] it was shown that these random codes are essentially asymptotically optimal, both in expectation and with probability one. Specifically, in [29] it is proved that

$$
E\left[\ell_{n}^{*}\left(X_{1}^{n}\right)\right] \approx n R(D)+\log n \text { bits }
$$

but we also know [35] that any code operating at distortion level $D$ has expected code lengths at least as large as $\approx n R(D)+(1 / 2) \log n$ bits. Similarly, in [21] it was shown that no code operating at distortion level $D$ can outperform $\ell_{n}^{*}\left(X_{1}^{n}\right)$ by more than $2 \log n$ bits, eventually with probability one, and it was also shown that $\ell_{n}^{*}\left(X_{1}^{n}\right)$ is approximately competitively optimal (see [21, Corollary 2 and Proposition 3]).

Therefore, we interpret (23) as saying that the pointwise price paid for universality by a mixture codebook is approximately "( $1 / 2) \log n$ bits per degree of freedom." The converse recently proved in [28] indicates that this rate is asymptotically optimal.

b) Conditions for Universality: The results of Theorem 7 and Corollary 1 only hold for sources $P$ in $\mathcal{S}(D)$. The only essential restriction implied by this assumption is that the optimal reproduction distribution at distortion level $D$ is unique and has full support. Although this is, of course, not always the case, it is typically true for all low enough distortion levels, and it is true in several important special cases. For example, it is easily seen that this assumption is satisfied in the case of binary sources with respect to Hamming distortion; cf. [21, Example 2]. More generally, Yang and Zhang in [30], [28] give the following sufficient condition:

The matrix $\left(e^{\lambda \rho(a, \hat{a})}\right)_{a \in A, \hat{a} \in \hat{A}}$ is of full column rank, for all $\lambda<0 . \quad(*)$

In [30, Appendix A] it is shown that for any source $P$ satisfying $(*)$, the optimal reproduction distribution $Q^{*}$ is unique for all $D \in\left(0, D_{\max }\right)$. [Note that the direct coding theorems in [30], [28] are stated for sources $P$ that satisfy (*) with $Q^{*}$ of full support; these conditions are apparently stronger than requiring $P \in \mathcal{S}(D)$.]

c) Pointwise Redundancy and Minimal Coding Variance: As discussed in remark a), the codes exhibited in Corollary 1 have code lengths that do not exceed the optimum $\ell_{n}^{*}\left(X_{1}^{n}\right)$ bits by more than $O(\log n)$ bits; cf. [21, Corollary 1]. Therefore, the mixture codebooks not only achieve the best rate, but their second-order performance is also optimal, in that their fluctuations universally achieve the "minimal coding variance" [21, p. 139] of the source that is being compressed. 


\section{Arbitrary Sources: The CoDES-MeAsures CORRESPONDENCE}

Before giving the proofs of Theorems 1-4, we state some preliminary lemmas that will be needed in the proofs of the direct coding theorems. The first lemma (given here without proof) describes a specific prefix-free code for the positive integers. It is based on iterating a simple idea of Elias [11].

Lemma 2. A Code for the Positive Integers: There exists a prefix-free code $C$ for the positive integers with associated length function $L$, such that, for all $k \geq 1$

$$
L(k) \leq \log k+\log ^{+} \log k+2 \log ^{+} \log ^{+} \log k+\gamma
$$

where $\log ^{+} x$ denotes the function $\log \max \{x, 1\}$, and $\gamma$ is some finite constant.

All our direct coding theorems will be proved using random coding arguments based on random codebooks as described in Section II-B. In the notation of that section, the next lemma establishes a precise relationship between the waiting time $W_{n}$ for a $D$-close match in the codebook and the probability $q_{n}=$ $Q_{n}\left(B\left(X_{1}^{n}, D\right)\right)$ of finding such a match.

Lemma 3. Waiting Times Bounds: In the notation of Section II-B:

i) if for some $x_{1}^{n} \in A^{n}$, the probability $q_{n} \triangleq Q_{n}\left(B\left(x_{1}^{n}, D\right)\right)$ is nonzero, then for any $\epsilon>0$

$$
\operatorname{Pr}\left\{\log \left[\left(W_{n}-1\right) q_{n}\right] \geq \epsilon \mid X_{1}^{n}=x_{1}^{n}\right\} \leq e^{-2^{c}} ;
$$

ii) there is a universal constant $K<\infty$ such that

$$
E\left\{\log \left[W_{n} Q_{n}\left(B\left(X_{1}^{n}, D\right)\right)\right]\right\} \leq K, \quad \text { for all } n \geq 1
$$

where for each $n$ the expectation is taken over the message $X_{1}^{n}$ as well as over the random codebook.

Proof of Lemma 3: Fix $x_{1}^{n}$ such that

$$
q_{n}=Q_{n}\left(B\left(x_{1}^{n}, D\right)\right)>0 .
$$

Then, conditional on $X_{1}^{n}=x_{1}^{n}$, the distribution of $W_{n}$ is geometric with parameter $q_{n}$ and

$$
\begin{aligned}
& \operatorname{Pr}\left\{\log \left[\left(W_{n}-1\right) q_{n}\right] \geq \epsilon \mid X_{1}^{n}=x_{1}^{n}\right\} \\
& \quad=\operatorname{Pr}\left\{W_{n} \geq 2^{\epsilon} / q_{n}+1 \mid X_{1}^{n}=x_{1}^{n}\right\} \leq\left(1-q_{n}\right)^{2^{c} / q_{n}} \leq e^{-2^{c}}
\end{aligned}
$$

where the last step follows from the simple inequality

$$
(1-x)^{1 / x} \leq e^{-1}, \quad \text { for } x \in(0,1] \text {. }
$$

This proves part i) of the lemma. For part ii), let $G_{n}$ denote the event

$$
G_{n}=\left\{W_{n} \neq 1 \text { and } Q_{n}\left(B\left(X_{1}^{n}, D\right)\right)>0\right\}
$$

and write $q_{n}$ for the (random) probability $Q_{n}\left(B\left(X_{1}^{n}, D\right)\right.$ ). Then we can bound

$$
\begin{aligned}
& E\left\{\log \left(W_{n} q_{n}\right)\right\} \\
& \quad \leq E\left\{\left[\log \left(W_{n} q_{n}\right)\right] \rrbracket_{G_{n}}\right\} \\
& \quad \leq E\left\{\left[\log \left(\left(W_{n}-1\right) q_{n}\right)\right] \rrbracket_{G_{n}}\right\}+1 \\
& \quad \leq \sum_{j \geq 0} \operatorname{Pr}\left\{\left[\log \left(\left(W_{n}-1\right) q_{n}\right)\right] \rrbracket_{G_{n}} \geq j\right\}+1 \\
& \quad \stackrel{\text { (a) }}{=} E_{\mathbb{P}}\left[\rrbracket_{G_{n}} \sum_{j \geq 0} \operatorname{Pr}\left\{\log \left(\left(W_{n}-1\right) q_{n}\right) \geq j \mid X_{1}^{\infty}\right\}\right]+1 \\
& \quad \stackrel{\text { (b) }}{\leq} \sum_{j \geq 0} e^{-2^{j}}+1 \triangleq K
\end{aligned}
$$

where (a) follows by Fubini's theorem, (b) follows from part i) of the lemma, and where for any event $G$, by $\rrbracket_{G}$ we denote its indicator function.

\section{A. Proof of Theorem 1}

For part i), given a code $C_{n}=\left(B_{n}, \phi_{n}, \psi_{n}, \ell_{n}\right)$, let $L_{n}: B_{n} \rightarrow \mathbb{N}$ be the length function of the uniquely decodable map $\psi_{n}$ so that $\ell_{n}\left(x_{1}^{n}\right)=L_{n}\left(\phi_{n}\left(x_{1}^{n}\right)\right)$. Define

$$
Q_{n}\left(y_{1}^{n}\right) \triangleq \begin{cases}2^{-L_{n}\left(y_{1}^{n}\right)}, & \text { if } y_{1}^{n} \in B_{n} \\ 0, & \text { otherwise. }\end{cases}
$$

Then for any $x_{1}^{n} \in A^{n}$

$$
\begin{aligned}
\ell_{n}\left(x_{1}^{n}\right)=L_{n}\left(\phi_{n}\left(x_{1}^{n}\right)\right)=-\log & Q_{n}\left(\phi_{n}\left(x_{1}^{n}\right)\right) \\
& \geq-\log Q_{n}\left(B\left(x_{1}^{n}, D\right)\right) \quad \text { bits }
\end{aligned}
$$

where the inequality follows from the fact that $C_{n}$ operates at distortion level $D$. Since $\psi_{n}$ is uniquely decodable, Kraft's inequality implies that $Q_{n}$ is a subprobability measure. If it is a probability measure we are done; otherwise, the above argument can be repeated with the measure $Q_{n}^{\prime}=Z^{-1} Q_{n}$ in place of $Q_{n}$, where $Z$ is the normalizing constant

$$
Z=\sum_{y_{1}^{n} \in B_{n}} 2^{-L_{n}\left(y_{1}^{n}\right)} \leq 1 .
$$

The direct coding theorems of parts ii) and iii) are based on a random coding argument. Given a sequence of measures $\left\{Q_{n}\right\}$, for each $n \geq 1$ generate a random codebook $\left\{Y_{1}^{n}(i) ; i \geq 1\right\}$ as described in Section II-B. These codebooks are assumed to be available both to the encoder and decoder, and the following coding scheme is adopted. If the waiting time $W_{n}$ is finite, then $X_{1}^{n}$ is described (with distortion $D$ or less) by describing $W_{n}$ to the decoder, using the code from Lemma 2. If $W_{n}=\infty$, then we describe $X_{1}^{n}$ using the quantizer $q^{(n)}$ provided by the condition (WQC). Writing $\mu_{n}$ for the distribution of $q^{(n)}\left(X_{1}^{n}\right)$ on $B_{n}$, this description can be given using at most

$$
\left\lceil-\log \mu_{n}\left(q^{(n)}\left(X_{1}^{n}\right)\right)\right\rceil \text { bits. }
$$

Finally, a 1-bit flag is added to tell the decoder which of the two cases ( $W_{n}<\infty$ or $W_{n}=\infty$ ) occurred. This code clearly operates at distortion level $D$, and its overall description length $\ell_{n}$ has

$\ell_{n}\left(X_{1}^{n}\right) \leq\left\{\begin{aligned} \log W_{n}+\log ^{+} \log W_{n} & \\ +2 \log ^{+} \log ^{+} \log W_{n}+\gamma+1, & \text { if } W_{n}<\infty \\ \left\lceil-\log \mu_{n}\left(q^{(n)}\left(X_{1}^{n}\right)\right)\right\rceil+1, & \text { if } W_{n}=\infty\end{aligned}\right.$

For part ii), since the sequence $\left\{Q_{n}\right\}$ is admissible, for $\mathbb{P}$-almost every realization of the source the probability

$$
Q_{n}\left(B\left(X_{1}^{n}, D\right)\right) \geq 2^{-n(R+\epsilon)}>0
$$

eventually, and therefore the waiting times $W_{n}$ will be finite eventually with probability 1 (with respect to both the source and the codebook distribution). Therefore,

$$
\begin{array}{r}
\ell_{n}\left(X_{1}^{n}\right) \leq \log W_{n}+\log ^{+} \log W_{n}+2 \log ^{+} \log ^{+} \log W_{n} \\
+\gamma+1 \text { eventually, w.p. } 1 .
\end{array}
$$


Write, as before, $q_{n}$ for the (random) probability $Q_{n}\left(B\left(X_{1}^{n}, D\right)\right)$. Then, for $\mathbb{P}$-almost every realization of the source, for $n$ large enough we can apply part i) of Lemma 3 with $\epsilon=\log [2 \log n]$ to get

$$
\begin{aligned}
\operatorname{Pr}\left\{\log \left[\left(W_{n}-1\right) q_{n}\right] \geq \log [2 \log n] \mid X_{1}^{n}\right. & \left.=x_{1}^{n}\right\} \\
& \leq e^{-2 \log n} \leq n^{-2} .
\end{aligned}
$$

The Borel-Cantelli lemma implies that

$$
\log \left[\left(W_{n}-1\right) q_{n}\right] \leq \log \log n+1 \quad \text { eventually, w.p. } 1
$$

and hence

$$
\log \left[W_{n} q_{n}\right] \leq \log \log n+2 \text { eventually, w.p. } 1 .
$$

From the admissibility of $\left\{Q_{n}\right\}$ and (26) we also have

$$
\log W_{n} \leq 2 n R \quad \text { eventually, w.p. } 1
$$

and combining (25)-(27)

$$
\begin{aligned}
\ell_{n}\left(X_{1}^{n}\right) \leq & -\log q_{n}+\log \log n+\log (2 n R) \\
& +2 \log \log (2 n R)+\gamma+3 \\
\leq & -\log q_{n}+\log n+3 \log \log n+[\gamma+3 \log R+6] \\
& \quad \text { eventually, w.p. } 1 .
\end{aligned}
$$

This proves part ii) of the theorem with the constant term equal to $(\gamma+3 \log R+6)$.

Turning to part iii), we note that the assumption that $\left\{Q_{n}\right\}$ is admissible in expectation implies that for all $\epsilon>0$ sufficiently small there is a finite (nonrandom) $N$ such that, for all $n \geq N$

and

$$
q_{n}=Q_{n}\left(B\left(X_{1}^{n}, D\right)\right)>0 \quad \text { w.p. } 1
$$

$$
E\left[-\log Q_{n}\left(B\left(X_{1}^{n}, D\right)\right)\right] \leq 2 n(R-\epsilon) .
$$

In particular, the bound (25) holds with probability one for all $n \geq N$, and, therefore,

$$
\begin{aligned}
E\left[\ell_{n}\left(X_{1}^{n}\right)\right] & \leq E\left[\log W_{n}\right]+E\left[\log ^{+} \log W_{n}\right] \\
& +2 E\left[\log ^{+} \log ^{+} \log W_{n}\right]+\gamma+1, \quad \text { for } n \geq N .
\end{aligned}
$$

Replacing all the $\left(\log W_{n}\right)$ terms above by $\left[\log \left(W_{n} q_{n}\right)-\right.$ $\left.\log q_{n}\right]$, using part ii) of Lemma 3 , and applying Jensen's inequality, implies that for all $n \geq N$

$$
\begin{aligned}
E\left[\ln _{n}\left(X_{1}^{n}\right)\right] \leq E[ & \left.-\log q_{n}\right]+\log \left[K+E\left(-\log q_{n}\right)\right] \\
& +2 \log \log \left[K+E\left(-\log q_{n}\right)\right]+\gamma+K+1 .
\end{aligned}
$$

Finally, using the bound (28) yields, for all $n \geq N$ large enough

$$
\begin{aligned}
E\left[\ell_{n}\left(X_{1}^{n}\right)\right] \leq E\left[-\log q_{n}\right]+\log n+ & 2 \log \log n \\
+ & {[\gamma+K+3 \log R+4] . }
\end{aligned}
$$

This proves part iii) with the constant term equal to $(\gamma+K+$ $3 \log R+4)$ and concludes the proof.

Remark: The proof of the direct coding theorem in part ii) of Theorem 1 establishes that the bound claimed in the statement of the theorem holds with probability one with respect to both the source and the random codebook distribution. In other words, not only deterministic codes exist as claimed, but (almost) any realization of the random codes constructed will provide such a code.

\section{B. Proof of Theorem 2}

The coding scheme used here is different from the one in Theorem 1. Let

$$
\Delta_{n} \triangleq-\log Q_{n}\left(B\left(X_{1}^{n}, D\right)\right)-n R
$$

so that $Z_{n}=\left|\Delta_{n}\right|$, and define the events

$$
H_{n} \triangleq\left\{Z_{n} \leq B \sqrt{n} \log n\right\}
$$

and

$$
J_{n} \triangleq\left\{\log W_{n} \leq n R+\Delta_{n}+\log \log n+2\right\} .
$$

If $H_{n}$ and $J_{n}$ both occur, then $W_{n}$ is described in two steps. First, we describe $\left\lceil\Delta_{n}\right\rceil$ using no more than

$$
\begin{aligned}
& \lceil\log (2 B \sqrt{n} \log n+1)\rceil \\
& \leq \frac{1}{2} \log n+\log \log n+\log B+3 \text { bits }
\end{aligned}
$$

and then describe $W_{n}$ itself using no more than

$$
\begin{aligned}
n R+\Delta_{n} & +\log \log n+3 \\
& =-\log Q_{n}\left(B\left(X_{1}^{n}, D\right)\right)+\log \log n+3 \text { bits. }
\end{aligned}
$$

If either $H_{n}$ or $J_{n}$ fails, then $X_{1}^{n}$ is described without coding, using the quantizer $q^{(n)}$ provided by (WQC). After adding a 1-bit flag to indicate which of the two methods was used, from (24), (29), and (30), the overall description length $\ell_{n}$ of this code has

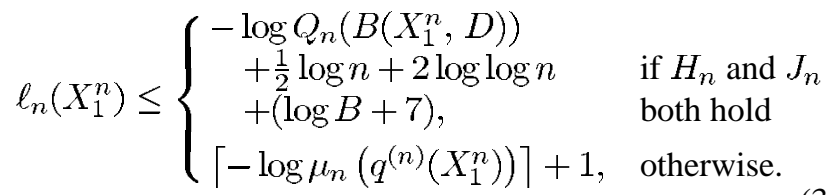

For part i), assumption (11) and the admissibility of $\left\{Q_{n}\right\}$ imply that both $H_{n}$ and $J_{n}$ hold eventually with probability 1 (see the derivation of (26) above), thereby proving part i) with the constant term being equal to $(\log B+7)$.

For part ii) of the theorem, we first need to bound the probability that $J_{n}$ fails. With $q_{n}=Q_{n}\left(B\left(X_{1}^{n}, D\right)\right)$, since $\left\{Q_{n}\right\}$ is admissible in expectation, for all $n$ large enough $q_{n}>0$ with probability one, and

$$
\begin{aligned}
\operatorname{Pr}\left\{J_{n} \text { fails }\right\} & =\operatorname{Pr}\left\{\log \left[W_{n} q_{n}\right]>\log (4 \log n)\right\} \\
& \leq \operatorname{Pr}\left\{\left(W_{n}-1\right) q_{n}>2 \log n\right\} \stackrel{\text { (a) }}{\leq} \frac{1}{n^{2}}
\end{aligned}
$$

where (a) follows by part i) of Lemma 3. Therefore, letting $F_{n}$ denote the event that either $H_{n}$ or $J_{n}$ fails, for $n$ large enough we have

$$
\operatorname{Pr}\left\{F_{n}\right\} \leq \frac{C+1}{n^{q}} .
$$

Taking expectations of both sides of (31) we get

$$
\begin{aligned}
E\left[\ell_{n}\left(X_{1}^{n}\right)\right] & \leq \\
\leq & E\left[-\log Q_{n}\left(B\left(X_{1}^{n}, D\right)\right)\right] \\
& +\frac{1}{2} \log n+2 \log \log n+(\log B+7) \\
& +E\left\{\rrbracket_{F_{n}}\left[-\log \mu_{n}\left(q^{(n)}\left(X_{1}^{n}\right)\right)\right]\right\}+2 \operatorname{Pr}\left\{F_{n}\right\} .
\end{aligned}
$$


Applying Hölder's inequality to the last expectation above, using bound (32), and recalling the bound from the condition $(p \mathrm{SQC})$, yields

$$
\begin{aligned}
& E\left\{\mathbb{1}_{F_{n}}\left[-\log \mu_{n}\left(q^{(n)}\left(X_{1}^{n}\right)\right)\right]\right\}+2 \operatorname{Pr}\left\{F_{n}\right\} \\
& \leq M_{p}(C+1)^{1 / q}+1, \quad \text { eventually. }
\end{aligned}
$$

This completes the proof of part ii) pf Theorem 2 with the constant term being $M_{p}(C+1)^{1 / q}+\log B+8$.

\section{Proofs of Theorems 3 and 4}

Lemma 1 immediately implies that $K_{n}(D) \geq R_{n}(D)$. Given a code $\left(C_{n}, \ell_{n}\right)$, define a probability measure $Q_{n}^{\prime}$ as in the proof of Theorem 1, and notice that

$$
E\left[\ell_{n}\left(X_{1}^{n}\right)\right] \geq E\left[-\log Q_{n}^{\prime}\left(B\left(X_{1}^{n}, D\right)\right)\right] \geq K_{n}(D) .
$$

Part ii) of Theorem 3 and part i) of Theorem 4 follow from the Kuhn-Tucker conditions given in [3] exactly as in [21, proof of Theorem 6]. Finally, part ii) of Theorem 4 is an immediate consequence of parts ii) and iii) of Theorem 1.

\section{Memoryless Sources: Mixture Codebooks AND REDUNDANCY}

In the notation of Section II-E, assume that $\left\{X_{n}\right\}$ is a stationary memoryless source with distribution $P$ on $A$, and write $\mathbb{P}$ for the distribution of the entire process. Let $\pi$ be a prior distribution on the simplex $\mathcal{P}$ of probability measures on $\hat{A}$. For each $n \geq 1$, the mixture distributions $M_{n}$ on $\hat{A}^{n}$ are defined as in (6).

\section{A. Proof of Theorem 6}

It suffices to show that for every $\epsilon>0$

$$
\frac{1}{n} \log \frac{Q_{n}^{*}\left(B\left(X_{1}^{n}, D\right)\right)}{M_{n}\left(B\left(X_{1}^{n}, D\right)\right)} \leq \epsilon \quad \text { eventually w.p. } 1
$$

or equivalently

$$
\frac{2^{n \epsilon} M_{n}\left(B\left(X_{1}^{n}, D\right)\right)}{Q_{n}^{*}\left(B\left(X_{1}^{n}, D\right)\right)} \geq 1, \quad \text { eventually w.p. } 1 .
$$

For $\epsilon>0$, define the neighborhoods $N\left(Q^{*}, \epsilon\right)$ as in (20), and assume that $\pi\left\{N\left(Q^{*}, \epsilon\right)\right\}>0$ for all $\epsilon>0$ (we deal with the case of $\pi$ having a positive density at the end).

Observe that

$$
\begin{aligned}
& \frac{2^{n \epsilon} M_{n}\left(B\left(X_{1}^{n}, D\right)\right)}{Q_{n}^{*}\left(B\left(X_{1}^{n}, D\right)\right)} \\
& =2^{n \epsilon} \int_{Q \in \mathcal{P}} \frac{Q^{n}\left(B\left(X_{1}^{n}, D\right)\right)}{Q_{n}^{*}\left(B\left(X_{1}^{n}, D\right)\right)} d \pi(Q) \\
& \quad \geq 2^{n \epsilon} \int_{N\left(Q^{*}, \epsilon\right)} \frac{Q^{n}\left(B\left(X_{1}^{n}, D\right)\right)}{Q_{n}^{*}\left(B\left(X_{1}^{n}, D\right)\right)} d \pi(Q) \\
& =\int_{N\left(Q^{*}, \epsilon\right)} 2^{n\left[\epsilon-\frac{1}{n} \log \frac{\left.Q_{n\left(2\left(B\left(X_{1}^{n}, D\right)\right)\right.}^{Q^{n}\left(B\left(X_{1}^{1}, D\right)\right\}}\right]}{2} d \pi(Q) .\right.}
\end{aligned}
$$

From (17) we know that for every $Q$, as $n \rightarrow \infty$

$$
\begin{aligned}
r_{n}\left(X_{1}^{n}\right) & \triangleq \frac{1}{n} \log \frac{Q_{n}^{*}\left(B\left(X_{1}^{n}, D\right)\right)}{Q^{n}\left(B\left(X_{1}^{n}, D\right)\right)} \\
& \rightarrow R(P, Q, D)-R(D) \quad \text { with } \mathbb{P} \text {-prob.1 }
\end{aligned}
$$

therefore, by Fubini's theorem, for $\mathbb{P}$-almost every realization $x_{1}^{\infty}$ of the source and for $\pi$-almost every $Q \in N\left(Q^{*}, \epsilon\right)$

$$
r_{n}\left(x_{1}^{n}\right) \rightarrow R(P, Q, D)-R(D)<\epsilon .
$$

Fix any one of the (almost all) $x_{1}^{\infty}$ such that (35) holds. By Fatou's lemma and the fact that $\pi\left\{N\left(Q^{*}, \epsilon\right)\right\}>0$ it follows that

$$
\begin{aligned}
\liminf _{n \rightarrow \infty} \int_{N\left(Q^{*}, \epsilon\right)} 2^{n\left(\epsilon-r_{n}\left(x_{1}^{n}\right)\right)} d \pi(Q) \\
\geq \int_{N\left(Q^{*}, \epsilon\right)} \liminf _{n \rightarrow \infty} 2^{n\left(\epsilon-r_{n}\left(x_{1}^{n}\right)\right)} d \pi(Q)=\infty
\end{aligned}
$$

and combining this with (34) implies (33) as required.

Finally, we claim that, if $\pi$ has a density $p(Q)$ with respect to Lebesgue measure on $\mathcal{P}$ and $p(Q)$ is strictly positive in a neighborhood of $Q^{*}$, then $\pi\left\{N\left(Q^{*}, \epsilon\right)\right\}>0$ for all $\epsilon>0$ small enough. Note that, since $p(Q)>0$ in a neighborhood of $Q^{*}$, it suffices to show that the neighborhoods $N\left(Q^{*}, \epsilon\right)$ have positive Lebesgue measure. Recall that by the representation of $R(P, Q, D)$ in [21, Proposition 2], $R(P, Q, D)$ is convex as a function of $Q$. Since $R\left(P, Q^{*}, D\right)=R(D)<\infty$, by the definition of $R(P, Q, D)$ it follows that it is finite for all $Q$ with support at least as large as the support of $Q^{*}$. Let $\mathcal{M}\left(Q^{*}\right)$ denote the set of all such $Q$

$$
\mathcal{M}\left(Q^{*}\right) \triangleq\left\{Q \in \mathcal{P}: \quad \operatorname{support}(Q) \supseteq \operatorname{support}\left(Q^{*}\right)\right\} .
$$

Since $\mathcal{M}\left(Q^{*}\right)$ is locally simplicial, $R(P, Q, D)$ is upper-semicontinuous on $\mathcal{M}\left(Q^{*}\right)$ (cf. [24, Theorem 10.2]). Therefore, the neighborhoods $N\left(Q^{*}, \epsilon\right)$ contain nonempty open sets and hence have positive Lebesgue measure. This proves the claim and completes the proof of the theorem.

\section{B. Technical Properties}

Following the notation of Section II-E, we let $\mathcal{P}$ denote the simplex of probability distributions on $\hat{A}$. Let $\hat{A}=$ $\left\{\hat{a}_{0}, \hat{a}_{1}, \ldots, \hat{a}_{d}\right\}$. For the sake of rigor, we need to introduce a simple parametrization of $\mathcal{P}$. We identify $\mathcal{P}$ with the $d$-dimensional subset of $\mathbb{R}^{d}$

$$
\Theta=\left\{\theta=\left(\theta_{1}, \theta_{2}, \ldots, \theta_{d}\right) \in[0,1]^{d}: \sum_{i=1}^{d} \theta_{i} \leq 1\right\}
$$

via the $1-1$ correspondence $Q \in \mathcal{P} \leftrightarrow \theta \in \Theta$

$$
\begin{aligned}
& Q\left(\hat{a}_{i}\right)=\theta_{i}, \quad i=1,2, \ldots, d \\
& Q\left(\hat{a}_{0}\right)=1-\sum_{i=1}^{d} \theta_{i} .
\end{aligned}
$$

We often write $Q_{\theta}$ for the distribution in $\mathcal{P}$ corresponding to $\theta \in \Theta$. 
Let $\pi$ be a prior distribution on $\Theta$ (or, equivalently, on $\mathcal{P}$ ). From now on, we also assume that $\pi$ has a strictly positive, continuous density

$$
p(\theta)=\frac{d \pi(\theta)}{d \theta}, \quad \theta \in \Theta
$$

with respect to Lebesgue measure on $\Theta$.

Throughout this section, we fix a $D>0$ and a source distribution $P \in \mathcal{S}(D)$ (recall (21)). Following [21], we define, for any $Q \in \mathcal{P}$

$$
\begin{aligned}
& D_{\min }^{P, Q} \triangleq E_{P}\left[\min _{y \in \operatorname{support}(Q)} \rho(X, y)\right] \\
& D_{\max }^{P, Q} \triangleq E_{P \times Q}[\rho(X, Y)]
\end{aligned}
$$

and for all $\lambda<0$ and $x \in A$

$$
\begin{aligned}
\Lambda_{P, Q}(\lambda) & \triangleq E_{P}\left\{\log _{e} E_{Q}\left[e^{\lambda \rho(X, Y)}\right]\right\} \\
\Lambda_{x}(Q, \lambda) & \triangleq \log _{e} E_{Q}\left[e^{\lambda \rho(x, Y)}\right] .
\end{aligned}
$$

By [21, Lemma 1], for any $Q=Q_{\theta} \in \mathcal{P}$ and any $D \in$ $\left(D_{\min }^{P, Q_{\theta}}, D_{\max }^{P, Q_{\theta}}\right)$ there exists a unique $\lambda=\lambda_{\theta}<0$ such that

$$
\left.\Lambda_{P, Q}^{\prime}\left(\lambda_{\theta}\right) \triangleq \frac{\partial}{\partial \lambda}\left[\Lambda_{P, Q}(\lambda)\right]\right|_{\lambda=\lambda_{\theta}}=D
$$

From [21, Lemma 1 and Proposition 1] we have

$$
R_{e}\left(P, Q_{\theta}, D\right)=\lambda_{\theta} D-\Lambda_{P, Q}\left(\lambda_{\theta}\right)
$$

where $R_{e}(P, Q, D)$ is the same as $R(P, Q, D)$ but in nats rather than bits

$$
R_{e}(P, Q, D) \triangleq\left(\log _{e} 2\right) R(P, Q, D) .
$$

Now let $\theta^{*}$ correspond to the optimal reproduction distribution $Q^{*}=Q_{\theta^{*}}$ for the source distribution $P$ at distortion level $D$. Recall [21, Proposition 2] that whenever $D \in\left(0, D_{\max }\right)$, we also have $D \in\left(D_{\min }^{P, Q^{*}}, D_{\max }^{P, Q^{*}}\right)$. Therefore, writing $\lambda^{*}=\lambda_{\theta^{*}}$

$$
R_{e}(D)=R_{e}\left(P, Q^{*}, D\right)=\lambda^{*} D-\Lambda_{P, Q^{*}}\left(\lambda^{*}\right)
$$

where $R_{e}(D)=\left(\log _{e} 2\right) R(D)$ denotes the rate-distortion function in nats.

Lemma 4. Differentiability Properties: Let $D>0$ and $P \in$ $\mathcal{S}(D)$. Write $Q^{*}=Q_{\theta^{*}}$ for the optimal reproduction distribution for $P$ at distortion level $D$.

i) For each $x \in A$, the function $\Lambda_{x}\left(Q_{\theta}, \lambda_{\theta}\right)$ is twice differentiable in $\theta$ on a neighborhood of $\theta^{*}$.

ii) $R_{e}\left(P, Q_{\theta}, D\right)$ is twice differentiable in $\theta$ on a neighborhood of $\theta^{*}$.

Proof of Lemma 4: For $\lambda<0$ and $\theta$ in the interior of $\Theta$ (corresponding to $Q_{\theta}$ in the interior of the simplex $\mathcal{P}$ ), define

$$
\Psi_{1}(\theta, \lambda)=\frac{\partial}{\partial \lambda}\left[\Lambda_{P, Q_{\theta}}(\lambda)\right]-D .
$$

By the definitions of $\lambda_{\theta}$ and $\lambda^{*}$ we have

$$
\Psi_{1}\left(\theta, \lambda_{\theta}\right)=\Psi_{1}\left(\theta^{*}, \lambda^{*}\right)=0 .
$$

It is easy to see that $\Psi_{1}$ is twice differentiable in either of its arguments, and, moreover, the derivative

$$
\frac{\partial \Psi_{1}(\theta, \lambda)}{\partial \lambda}=\Lambda_{P, Q_{\theta}}^{\prime \prime}(\lambda)
$$

exists and is strictly positive as long as $D_{\min }^{P, Q_{\theta}}$ is strictly smaller than $D_{\max }^{P, Q_{\theta}}$ (see [21, Lemma 1]). But since $P$ and $Q_{\theta}$ both have full support, our assumption that $D_{\max }>0$ implies that this is always the case. Thus, by the implicit function theorem, $\lambda_{\theta}$ is differentiable in $\theta$ on a neighborhood of $\theta^{*}$, and

$$
\frac{\partial \lambda_{\theta}}{\partial \theta_{i}}=-\left.\left[\Lambda_{P, Q_{\theta}}^{\prime \prime}\left(\lambda_{\theta}\right)\right]^{-1} \frac{\partial \Psi_{1}(\theta, \lambda)}{\partial \theta_{i}}\right|_{\lambda=\lambda_{\theta}} .
$$

From this expression and from the differentiability of $\Psi_{1}$ it immediately follows that $\lambda_{\theta}$ is in fact twice differentiable in $\theta$ on a neighborhood of $\theta^{*}$, and since

$$
\Lambda_{x}\left(Q_{\theta}, \lambda_{\theta}\right)=\log _{e} \sum_{y \in \hat{A}}\left[Q_{\theta}(y) e^{\lambda_{\theta} \rho(x, Y)}\right]
$$

it follows that, for any fixed $x \in A, \Lambda_{x}\left(Q_{\theta}, \lambda_{\theta}\right)$ is also twice differentiable in $\theta$ in a neighborhood of $\theta^{*}$. This proves part i) of the lemma.

Recall the expression for $R_{e}(P, Q, D)$ in (36) and define, for $R>0$ and $\theta$ in the interior of $\Theta$

$$
\Psi_{2}(\theta, R)=R-\lambda_{\theta} D+\Lambda_{P, Q_{\theta}}\left(\lambda_{\theta}\right) .
$$

By the discussion preceding the statement of the lemma

$$
\Psi_{2}\left(\theta^{*}, R_{e}(D)\right)=\Psi_{2}\left(\theta, R_{e}\left(P, Q_{\theta}, D\right)\right)=0 .
$$

Also, by the preceding arguments, $\Psi_{2}$ is twice differentiable in either of its arguments and

$$
\frac{\partial \Psi_{2}(\theta, R)}{\partial R}=1 \neq 0 .
$$

Therefore, by the implicit function theorem $R_{e}\left(P, Q_{\theta}, D\right)$ is differentiable in $\theta$ on a neighborhood of $\theta^{*}$ and

$$
\frac{\partial R_{e}\left(P, Q_{\theta}, D\right)}{\partial \theta_{i}}=-\left.\frac{\partial \Psi_{2}(\theta, R)}{\partial \theta_{i}}\right|_{R=R\left(P, Q_{\theta}, D\right)} .
$$

This, together with the definition $\Psi_{2}(\theta, R)$ and the differentiability of $\lambda_{\theta}$ already proved, imply that $R_{e}\left(P, Q_{\theta}, D\right)$ is twice differentiable, proving part ii) of the lemma.

\section{Proof of Theorem 7}

The outline of the proof is similar to that of the corresponding lossless result in [6] and it heavily relies on the precise asymptotics for $Q_{n}^{*}\left(B\left(X_{1}^{n}, D\right)\right)$ developed in [9] and [29], so our notation follows closely the notation in [6], [9], [29].

Let $D>0$ be given, and choose and fix a source distribution $P \in \mathcal{S}(D)$ with a corresponding optimal reproduction distribution $Q^{*}=Q_{\theta^{*}}$. According to Lemma 4 we can define

$$
\begin{aligned}
S_{n} & \triangleq S_{n}\left(\theta^{*}, \lambda^{*} ; X_{1}^{n}\right) \\
& \left.\triangleq \frac{1}{\sqrt{n}} \sum_{i=1}^{n} \nabla_{\theta}\left[\Lambda_{X_{i}}\left(Q_{\theta}, \lambda_{\theta}\right)-\Lambda_{P, Q_{\theta}}\left(\lambda_{\theta}\right)\right]\right|_{\theta=\theta^{*}}
\end{aligned}
$$

where $\lambda^{*}=\lambda_{\theta^{*}}$ is chosen so that (37) holds. Similarly, Lemma 4 guarantees the existence of the matrix of partial derivatives

$$
\left.J \triangleq \frac{\partial^{2}}{\partial \theta^{2}} R_{e}\left(P, Q_{\theta}, D\right)\right|_{\theta=\theta^{*}} .
$$

Note that, since $P \in \mathcal{S}(D), J$ is positive-definite and hence invertible.

Since $S_{n}$ is a (normalized) partial sum of zero-mean, independent random vectors, the law of the iterated logarithm implies 
that each of its components is of order $O\left(\sqrt{\log _{e} \log _{e} n}\right)$ with probability one. Therefore, the quadratic form

$$
S_{n}^{t} J^{-1} S_{n}=O\left(\log _{e} \log _{e} n\right) \quad \text { w.p. } 1 .
$$

Choosing a $d_{0}>d$, we define a "perturbed" version of $\theta^{*}$ as

$$
\hat{\theta}=\theta^{*}+\frac{1}{\sqrt{n}} J^{-1} S_{n} \cdot \|_{\left\{S_{n}^{ \pm} J^{-1} S_{n} \leq d_{0}\right\}} \cdot
$$

Let $\delta_{n} \triangleq \sqrt{\frac{d_{0}}{n}}, n \geq 1$, and define a sequence of neighborhoods centered at $\hat{\theta}$

$$
\hat{N}_{\delta_{n}} \triangleq\left\{\theta \in \Theta:\|\theta-\hat{\theta}\| \leq \delta_{n}\right\}
$$

where, throughout the proof, $\|\cdot\|$ denotes the $L^{2}$-norm with respect to the matrix $J$

$$
\|\xi\| \triangleq \sqrt{\xi^{t} J \xi}, \quad \xi \in \mathbb{R}^{d} .
$$

Similarly define the neighborhoods

$$
N_{\delta_{n}}=\left\{\theta \in \Theta:\left\|\theta-\theta^{*}\right\| \leq \delta_{n}\right\}
$$

and note that

$$
\hat{N}_{\delta_{n}} \subset N_{2 \delta_{n}}
$$

Next we obtain an upper bound on the quantity $-\frac{n}{2}\|\theta-\hat{\theta}\|^{2}$. Using the definition of $\hat{\theta}$, we expand $\|\theta-\hat{\theta}\|^{2}$ as

$$
\begin{aligned}
\| \theta- & \theta^{*} \|^{2}+\frac{1}{n} S_{n}^{t} J^{-1} S_{n}-\frac{2}{\sqrt{n}}\left(\theta-\theta^{*}\right)^{t} S_{n} \\
& -\left\{\frac{1}{n} S_{n}^{t} J^{-1} S_{n}-\frac{2}{\sqrt{n}}\left(\theta-\theta^{*}\right)^{t} S_{n}\right\} \rrbracket_{\left\{S_{n}^{t} J^{-1} S_{n}>d_{0}\right\}} .
\end{aligned}
$$

Since on the event $\left\{S_{n}^{t} J^{-1} S_{n}>d_{0}\right\}$ we have $\hat{\theta}=\theta^{*}$, the Cauchy-Schwarz inequality implies that

$\sqrt{n}\left(\theta^{*}-\theta\right)^{t} S_{n} \rrbracket_{\left\{S_{n}^{t} J^{-1} S_{n}>d_{0}\right\}} \leq S_{n}^{t} J^{-1} S_{n}, \quad$ for $\theta \in \hat{N}_{\delta_{n 2}}$.

Combining (40) and (41) we have that, for all $\theta \in \hat{N}_{\delta_{n}}$

$-\frac{n}{2}\|\theta-\hat{\theta}\|^{2} \leq-\frac{n}{2}\left\|\theta-\theta^{*}\right\|^{2}+\sqrt{n}\left(\theta-\theta^{*}\right)^{t} S_{n}+S_{n}^{t} J^{-1} S_{n}$.

Now we are ready to examine the term $\log _{e}\left[\frac{Q_{n}^{*}\left(B\left(X_{1}^{n}, D\right)\right)}{M_{n}\left(B\left(X_{1}^{n}, D\right)\right)}\right]$. Let $\phi_{n}(\theta), n \geq 1$, denote the truncated normal density functions

$$
\phi_{n}(\theta)=\frac{1}{c_{n}} e^{-(n / 2)\|\theta-\hat{\theta}\|^{2}} \rrbracket_{\left\{\theta \in \hat{N}_{\varepsilon_{n}}\right\}}, \quad \theta \in \Theta
$$

where $c_{n}$ is the normalizing constant

$$
c_{n}=\int_{\hat{N}_{\delta_{n}}} e^{-(n / 2)\|\theta-\hat{\theta}\|^{2}} d \theta .
$$

Writing $Q_{n}^{*}=\left(Q^{*}\right)^{n}$ and $Q_{\theta}^{n}=\left(Q_{\theta}\right)^{n}$, with probability one we have

$$
\begin{aligned}
\log _{e} & \frac{Q_{n}^{*}\left(B\left(X_{1}^{n}, D\right)\right) p\left(\theta^{*}\right)}{M_{n}\left(B\left(X_{1}^{n}, D\right)\right)} \\
= & -\log _{e} \int_{\Theta} \frac{Q_{\theta}^{n}\left(B\left(X_{1}^{n}, D\right)\right) p(\theta)}{Q_{n}^{*}\left(B\left(X_{1}^{n}, D\right)\right) p\left(\theta^{*}\right)} d \theta \\
\leq & -\log _{e} \int_{\hat{N}_{\delta_{n}}} \frac{Q_{\theta}^{n}\left(B\left(X_{1}^{n}, D\right)\right) p(\theta)}{Q_{n}^{*}\left(B\left(X_{1}^{n}, D\right)\right) p\left(\theta^{*}\right)} d \theta
\end{aligned}
$$

$$
\begin{aligned}
& =-\log _{e} \int_{\hat{N}_{\delta_{n}}} \frac{Q_{\theta}^{n}\left(B\left(X_{1}^{n}, D\right)\right)}{Q_{n}^{*}\left(B\left(X_{1}^{n}, D\right)\right)} \frac{p(\theta)}{p\left(\theta^{*}\right)} c_{n} \\
& \cdot e^{(n / 2)\|\theta-\hat{\theta}\|^{2}} \phi_{n}(\theta) d \theta \\
& \stackrel{(a)}{\leq} \int_{\hat{N}_{\varepsilon_{n}}}\left[-\log _{e} \frac{Q_{\theta}^{n}\left(B\left(X_{1}^{n}, D\right)\right)}{Q_{n}^{*}\left(B\left(X_{1}^{n}, D\right)\right)}-\frac{n}{2}\|\theta-\hat{\theta}\|^{2}\right. \\
& \left.-\log _{e} \frac{p(\theta)}{p\left(\theta^{*}\right)}\right] \phi_{n}(\theta) d \theta-\log _{e} c_{n} \\
& \stackrel{(\mathrm{b})}{\leq} \int_{\hat{N}_{\delta_{n}}}\left[-\log _{e} \frac{Q_{\theta}^{n}\left(B\left(X_{1}^{n}, D\right)\right)}{Q_{n}^{*}\left(B\left(X_{1}^{n}, D\right)\right)}-\frac{n}{2}\left\|\theta-\theta^{*}\right\|^{2}\right. \\
& \left.+\sqrt{n}\left(\theta-\theta^{*}\right)^{t} S_{n}\right] \phi_{n}(\theta) d \theta \\
& -\log _{e} c_{n}+O\left(\log _{e} \log _{e} n\right) \\
& \stackrel{\text { (c) }}{\leq} \int_{N_{2 \varepsilon_{n}}} \mid-\log _{e} \frac{Q_{\theta}^{n}\left(B\left(X_{1}^{n}, D\right)\right)}{Q_{n}^{*}\left(B\left(X_{1}^{n}, D\right)\right)}-\frac{n}{2}\left\|\theta-\theta^{*}\right\|^{2} \\
& +\sqrt{n}\left(\theta-\theta^{*}\right)^{t} S_{n} \mid e_{0} \phi_{n}^{*}(\theta) d \theta \\
& -\log _{e} c_{n}+O\left(\log _{e} \log _{e} n\right)
\end{aligned}
$$

where $\phi_{n}^{*}(\theta)$ is the $d$-dimensional normal density with mean $\theta^{*}$ and covariance matrix $(n J)^{-1}$. In (43), (a) follows by Jensen's inequality, (b) follows from the continuity of $p(\theta),(42)$, and (38), and (c) follows from (39) and the fact that

$$
\phi_{n}(\theta) \leq e_{0} \phi_{n}^{*}(\theta), \quad \text { for some constant } e_{0}>0, \theta \in \hat{N}_{\delta_{n}} .
$$

This inequality is easily derived from the bound in [6, eq. (5.7) p. 467].

Next we consider the integrand in (43). Appealing to [29, Corollary 1], for $\theta$ close enough to $\theta^{*}$

$$
\begin{aligned}
& -\log _{e} Q_{n}^{*}\left(B\left(X_{1}^{n}, D\right)\right) \\
& \quad=n R_{e}\left(\hat{P}_{n}, Q^{*}, D\right)+\frac{1}{2} \log _{e} n+O(1) \quad \text { w.p. } 1
\end{aligned}
$$

and

$$
\begin{aligned}
& -\log _{e} Q_{\theta}^{n}\left(B\left(X_{1}^{n}, D\right)\right) \\
& \quad=n R_{e}\left(\hat{P}_{n}, Q_{\theta}, D\right)+\frac{1}{2} \log _{e} n+O(1) \quad \text { w.p. } 1
\end{aligned}
$$

where $\hat{P}_{n}$ denotes the empirical distribution induced by $X_{1}^{n}$ on $A$. Moreover, a close examination of [29, proof of Corollary 1] reveals that, in our setting (where $A$ and $\hat{A}$ are finite and $\rho$ is bounded), the convergence in (44) is uniform for $\theta$ in a small enough compact neighborhood of $\theta^{*}$. Therefore, uniformly for $\theta \in N_{2 \delta_{n}}$

$$
\begin{aligned}
\log _{e} & \frac{Q_{n}^{*}\left(B\left(X_{1}^{n}, D\right)\right)}{Q_{\theta}^{n}\left(B\left(X_{1}^{n}, D\right)\right)} \\
= & n\left[R_{e}\left(\hat{P}_{n}, Q_{\theta}, D\right)-R_{e}\left(\hat{P}_{n}, Q^{*}, D\right)\right]+O(1) \quad \text { w.p. } 1 .
\end{aligned}
$$

To expand the right-hand side of (45) further, following [21] we define

$$
f_{\theta}(x)=-\Lambda_{x}\left(Q_{\theta}, \lambda_{\theta}\right)+\Lambda_{P, Q_{\theta}}\left(\lambda_{\theta}\right), \quad x \in A .
$$

Writing the difference $n\left[R_{e}\left(\hat{P}_{n}, Q_{\theta}, D\right)-R_{e}\left(\hat{P}_{n}, Q^{*}, D\right)\right]$ as

$$
\begin{aligned}
& n\left[R_{e}\left(\hat{P}_{n}, Q_{\theta}, D\right)-R_{e}\left(P, Q_{\theta}, D\right)\right] \\
& \quad-n\left[R_{e}\left(\hat{P}_{n}, Q^{*}, D\right)-R_{e}\left(P, Q^{*}, D\right)\right] \\
& \quad+n\left[R_{e}\left(P, Q_{\theta}, D\right)-R_{e}\left(P, Q^{*}, D\right)\right]
\end{aligned}
$$


we can apply [9, Theorem 3] to get that

$$
n\left[R_{e}\left(\hat{P}_{n}, Q_{\theta}, D\right)-R_{e}\left(\hat{P}_{n}, Q^{*}, D\right)\right]
$$

is equal to

$$
\begin{aligned}
\sum_{i=1}^{n}\left[f_{\theta}\left(X_{i}\right)-f_{\theta^{*}}\left(X_{i}\right)\right]+n[ & \left.R_{e}\left(P, Q_{\theta}, D\right)-R_{e}\left(P, Q^{*}, D\right)\right] \\
& +O\left(\log _{e} \log _{e} n\right) \text { w.p. 1. }
\end{aligned}
$$

[Note that we have actually used the sharper remainder term of order $\left(\log _{e} \log _{e} n\right)$, as identified in [21, Appendix VI].] Further, a careful look at [9, proof of Theorem 3] (see also the discussion in [10, proof of Theorem 18]) shows the convergence in [9, Theorem 3] is uniform for $\theta$ in a small compact neighborhood in $\theta^{*}$ (provided that $A$ and $\hat{A}$ are finite and $\rho$ is bounded). Therefore, convergence in (46) holds uniformly for $\theta \in N_{2 \delta_{n}}$.

Notice that in the above notation $S_{n}$ can be rewritten as

$$
S_{n}=\left.\frac{1}{\sqrt{n}} \sum_{i=1}^{n} \nabla_{\theta}\left[-f_{\theta}\left(X_{i}\right)\right]\right|_{\theta=\theta^{*}} .
$$

From the Taylor expansion of $f_{\theta}$ around $\theta^{*}$, and taking the maximum over all possible realizations for $X_{1}^{n}=x_{1}^{n} \in A^{n}$, we get

$$
\begin{aligned}
\sum_{i=1}^{n}\left(f_{\theta}\left(X_{i}\right)-f_{\theta^{*}}\left(X_{i}\right)\right)+\sqrt{n}\left(\theta-\theta^{*}\right)^{t} S_{n} & \\
& =n O\left(\left\|\theta-\theta^{*}\right\|^{2}\right) \quad \text { w.p. } 1 .
\end{aligned}
$$

Therefore, uniformly for $\theta \in N_{2} \delta_{n}$

$$
\sum_{i=1}^{n}\left(f_{\theta}\left(X_{i}\right)-f_{\theta^{*}}\left(X_{i}\right)\right)+\sqrt{n}\left(\theta-\theta^{*}\right)^{t} S_{n}=O(1) \quad \text { w.p. } 1 \text {. }
$$

Combining (46) and (47) uniformly for $\theta \in N_{2} \delta_{n}$, we can bound

$$
\begin{aligned}
\mid n & {\left[R_{e}\left(\hat{P}_{n}, Q_{\theta}, D\right)-R_{e}\left(\hat{P}_{n}, Q^{*}, D\right)\right]-\frac{n}{2}\left\|\theta-\theta^{*}\right\|^{2} } \\
& +\sqrt{n}\left(\theta-\theta^{*}\right)^{t} S_{n} \mid \\
\leq & \left|\sum_{i=1}^{n}\left(f_{\theta}\left(X_{i}\right)-f_{\theta^{*}}\left(X_{i}\right)\right)+\sqrt{n}\left(\theta-\theta^{*}\right)^{t} S_{n}\right| \\
& +n\left|R_{e}\left(P, Q_{\theta}, D\right)-R_{e}\left(P, Q^{*}, D\right)-\frac{1}{2}\left\|\theta-\theta^{*}\right\|^{2}\right| \\
& +O\left(\log _{e} \log _{e} n\right) \\
= & O\left(\log _{e} \log _{e} n\right) \quad \text { w.p. } 1
\end{aligned}
$$

where in the last step we have used the Taylor expansion of $R_{e}\left(P, Q_{\theta}, D\right)$ around $\theta^{*}$

$R_{e}\left(P, Q_{\theta}, D\right)-R_{e}\left(P, Q^{*}, D\right)=\frac{1}{2}\left\|\theta-\theta^{*}\right\|^{2}+o\left(\left\|\theta-\theta^{*}\right\|^{2}\right)$

(recall Lemma 4 and the definition of the norm $\|\cdot\|$ ). Substituting the bounds (45) and (48) in (43) we conclude that

$\log _{e} \frac{Q_{n}^{*}\left(B\left(X_{1}^{n}, D\right)\right)}{M_{n}\left(B\left(X_{1}^{n}, D\right)\right)} \leq-\log _{e} c_{n}+O\left(\log _{e} \log _{e} n\right) \quad$ w.p. 1.
Finally (recall that $J$ is positive definite), repeating the exact same argument that leads to [6, eq. (5.3)], by a comparison with a multivariate normal integral we can estimate

$$
-\log _{e} c_{n} \leq \frac{d}{2} \log _{e} n+O(1)
$$

thereby completing the proof.

\section{APPENDIX I}

\section{PROOF OF LEMMA 1}

The proof that $K_{n}(D)$ is no smaller than the right-hand side of the statement of the lemma follows exactly as in the proof of [21, Lemma 4], with the only difference that, if the infimum in the definition of $K_{n}(D)$ is not achieved, we can assume without loss of generality that $K_{n}(D)<\infty$ and choose $\tilde{Q}_{n}$ with

$$
E\left[-\log \widetilde{Q}_{n}\left(B\left(X_{1}^{n}, D\right)\right)\right] \leq K_{n}(D)+\epsilon .
$$

Repeating the argument in [21, Appendix 5] up to step (d), yields that $K_{n}(D) \geq \inf I\left(X_{1}^{n} ; Y_{1}^{n}\right)-\epsilon$ and letting $\epsilon \downarrow 0$ gives the desired inequality.

To prove the reverse inequality, let $\left(X_{1}^{n}, Y_{1}^{n}\right)$ be an arbitrary pair of random vectors as in the infimum in the statement of the lemma, write $\mu_{n}$ for the joint distribution of $\left(X_{1}^{n}, Y_{1}^{n}\right)$, let $Q_{n}$ denote the $Y_{1}^{n}$-marginal, and assume that $I\left(X_{1}^{n} ; Y_{1}^{n}\right)<\infty$. [Note that if no such $\left(X_{1}^{n}, Y_{1}^{n}\right) \sim \mu_{n}$ exists then the infimum equals $+\infty$ and we are done.] This guarantees the existence of all the Radon-Nikodym derivatives below (in particular recall [12, eq. (5.2.8)]), so that for $P_{n}$-almost every $x_{1}^{n}$

$$
\begin{aligned}
& \int d \mu_{n}\left(y_{1}^{n} \mid x_{1}^{n}\right) \log \frac{d \mu_{n}\left(y_{1}^{n} \mid x_{1}^{n}\right)}{d Q_{n}} \\
& \stackrel{(\text { a) }}{=}-\int_{B\left(x_{1}^{n}, D\right)} d \mu_{n}\left(y_{1}^{n} \mid x_{1}^{n}\right) \log \frac{d Q_{n}}{d \mu_{n}}\left(y_{1}^{n} \mid x_{1}^{n}\right) \\
& \quad \stackrel{\text { (b) }}{\geq}-\log Q_{n}\left(B\left(x_{1}^{n}, D\right)\right)
\end{aligned}
$$

where (a) follows from Fubini's theorem and the assumption that $\rho\left(X_{1}^{n}, Y_{1}^{n}\right) \leq D$ with $\mu_{n}$-probability one, and (b) follows from Jensen's inequality. Integrating both sides above with respect to $P_{n}$ yields

$$
\begin{aligned}
I\left(X_{1}^{n} ; Y_{1}^{n}\right) & =H\left(\mu_{n} \| P_{n} \times Q_{n}\right) \\
& \geq E\left[-\log Q_{n}\left(B\left(X_{1}^{n}, D\right)\right)\right] \geq K_{n}(D)
\end{aligned}
$$

and completes the proof.

\section{APPENDIX II \\ PROOF OUTLINE FOR THEOREM 5}

We assume that (for all $n$ ) the infimum in the definition of $K_{n}(D)$ is achieved by some probability measure $\tilde{Q}_{n}$ on $\hat{A}^{n}$; as explained in the remarks following Theorems 4 and 5 the general case is similar. The existence of $R(D)$ is well known [12]. For each $n \geq 1$, let $\mathcal{G}_{n}$ be the family of functions defined by (13). Condition (QC) implies that $K_{n}(D)<\infty$ and, therefore, each $\mathcal{G}_{n}$ is nonempty. Moreover, in the terminology of [17], it is straightforward to check that each $\mathcal{G}_{n}$ is log-convex and that the sequence $\left\{\mathcal{G}_{n}\right\}$ is additive. Then Kieffer's generalized ergodic 
theorem [17] implies (14) with $K(D)$ on the right-hand side, and also establishes the existence of

$$
K(D)=\lim _{n \rightarrow \infty} K_{n}(D)=\inf _{n \geq 1} K_{n}(D) .
$$

Before proving the equality $K(D)=R(D)$ we note that part i) of Theorem 4 and (14) imply (15), and similarly part ii) of Theorem 1 and (14) show the existence of codes achieving (15) with equality. This proves part ii) of Theorem 5.

Finally, we argue that $K(D)=R(D)$. From their definitions and Lemma 1 it immediately follows that $R(D) \leq K(D)<\infty$. Fatou's lemma applied to (15) implies that for any sequence of codes $\left\{\left(C_{n}, \ell_{n}\right)\right\}$ operating at distortion level $D$

$$
\liminf _{n \rightarrow \infty} \frac{1}{n} E\left[\ell_{n}\left(X_{1}^{n}\right)\right] \geq K(D) .
$$

But there are codes operating at distortion level $D$ that achieve the rate-distortion function in expectation. A close examination of the proofs in [12, Theorems 11.4.1 and 11.5.1] shows that for any $\epsilon>0$ and $\delta>0$ there are fixed-rate codes with asymptotic rate bounded above by $R(D-\delta)+\epsilon$ and with vanishing probability of encoding a source string with distortion greater than $D$. Therefore, using the quantizers $\left\{q^{(n)}\right\}$ provided by (QC) we can modify these codes to operate at distortion level $D$, with an additional cost $\epsilon$ in the rate. This and (49) imply that for all $\epsilon>0$ and $\delta>0$

$$
R(D-\delta)+2 \epsilon \geq K(D) .
$$

Since $R(D)$ is continuous when finite, we can let both $\epsilon$ and $\delta$ go to zero to get that indeed $R(D) \geq K(D)$.

\section{ACKNOWLEDGMENT}

The authors wish to thank M. Harrison for his comments on an earlier draft of this paper.

\section{REFERENCES}

[1] A. R. Barron, "Logically smooth density estimation," Ph.D. dissertation, Dept. Elec. Eng., Stanford Univ., Stanford, CA, 1985.

[2] A. R. Barron, J. Rissanen, and B. Yu, "The minimum description length principle in coding and modeling. (Information theory: 1948-1998)," IEEE Trans. Inform. Theory, vol. 44, pp. 2743-2760, Oct. 1998.

[3] R. Bell and T. M. Cover, "Game-theoretic optimal portfolios," Manag. Sci., vol. 34, no. 6, pp. 724-733, 1988.

[4] T. Berger, Rate Distortion Theory: A Mathematical Basis for Data Compression. Englewood Cliffs, NJ: Prentice-Hall, 1971.

[5] P. A. Chou, M. Effros, and R. M. Gray, "A vector quantization approach to universal noiseless coding and quantizations," IEEE Trans. Inform. Theory, vol. 42, pp. 1109-1138, July 1996.

[6] B. S. Clarke and A. R. Barron, "Information-theoretic asymptotics of Bayes methods," IEEE Trans. Inform. Theory, vol. 36, pp. 453-471, May 1990.

[7] T. M. Cover and J. A. Thomas, Elements of Information Theory. New York: Wiley, 1991.

[8] L. D. Davisson, "Universal noiseless coding," IEEE Trans. Inform. Theory, vol. IT-19, pp. 783-795, Nov. 1973.

[9] A. Dembo and I. Kontoyiannis, "The asymptotics of waiting times between stationary processes, allowing distortion," Ann. Appl. Probab., vol. 9, pp. 413-429, 1999.
[10] — - "Source coding, large deviations, and approximate pattern matching," IEEE Trans. Inform. Theory, vol. 48, pp. 1590-1615, June 2002.

[11] P. Elias, "Universal codeword sets and representations of the integers," IEEE Trans. Inform. Theory, vol. IT-21, pp. 194-203, Mar. 1975.

[12] R. M. Gray, Source Coding Theory. Boston, MA: Kluwer Academic, 1990.

[13] T. S. Han, "An information-spectrum approach to source coding theorems with a fidelity criterion," IEEE Trans. Inform. Theory, vol. 43, pp. 1145-1164, July 1997.

[14] —, Information-Spectrum Methods in Information Theory (in Japanese). Tokyo, Japan: Baifukan-Press, 1998.

[15] D. Ishii and H. Yamamoto, "The redundancy of universal coding with a fidelity criterion," IEICE Trans. Fundamentals, vol. E80-A, pp. 2225-2231, 1997.

[16] F. Kanaya and K. Nakagawa, "On the practical implication of mutual information for statistical decision making," IEEE Trans. Inform. Theory, vol. 37, pp. 1151-1156, July 1991.

[17] J. C. Kieffer, "An almost sure convergence theorem for sequences of random variables selected from log-convex sets," in Almost Everywhere Convergence, II (Evanston, IL, 1989). Boston, MA: Academic, 1991, pp. $151-166$.

[18] _ "Sample converses in source coding theory," IEEE Trans. Inform. Theory, vol. 37, pp. 263-268, Mar. 1991.

[19] I. Kontoyiannis, "An implementable lossy version of the Lempel-Ziv algorithm-Part I: Optimality for memoryless sources," IEEE Trans. Inform. Theory, vol. 45, pp. 2293-2305, Nov. 1999.

[20] _ - "Model selection via rate-distortion theory," in Proc. 34th Annu. Conf. Information Sciences and Systems, CISS 2000, Princeton, NJ, Mar. 2000, pp. WP8-7-WP8-11.

[21] - "Pointwise redundancy in lossy data compression and universal lossy data compression," IEEE Trans. Inform. Theory, vol. 46, pp. 136-152, Jan. 2000.

[22] T. Łuczak and W. Szpankowski, "A suboptimal lossy data compression algorithm based on approximate pattern matching," IEEE Trans. Inform. Theory, vol. 43, pp. 1439-1451, Sept. 1997.

[23] J. Rissanen, "Stochastic complexity," J. Roy. Statist. Soc. Ser. B, vol. 49, no. 3, pp. 223-239, 253-265, 1987. With discussion.

[24] R. T. Rockafellar, Convex Analysis. Princeton, NJ: Princeton Univ. Press, 1997, Reprint of the 1970 original, Princeton Paperbacks.

[25] C. E. Shannon, "Coding theorems for a discrete source with a fidelity criterion," in IRE Nat. Conv. Rec., 1959, pp. 142-163.

[26] Y. Steinberg and S. Verdú, "Simulation of random processes and ratedistortion theory," IEEE Trans. Inform. Theory, vol. 42, pp. 63-86, Jan. 1996.

[27] E.-h. Yang and J. C. Kieffer, "On the performance of data compression algorithms based upon string matching," IEEE Trans. Inform. Theory, vol. 44, pp. 47-65, Jan. 1998.

[28] E.-h. Yang and Z. Zhang, "The redundancy of source coding with a fidelity criterion-Part III: Coding at a fixed distortion level with unknown statistics," preprint.

[29] — "On the redundancy of lossy source coding with abstract alphabets," IEEE Trans. Inform. Theory, vol. 45, pp. 1092-1110, May 1999.

[30] - "The redundancy of source coding with a fidelity criterion-Part II: Coding at a fixed rate level with unknown statistics," IEEE Trans. Inform. Theory, vol. 47, pp. 126-145, Jan. 2001.

[31] B. Yu and T. P. Speed, "A rate of convergence result for a universal $D$-semifaithful code," IEEE Trans. Inform. Theory, vol. 39, pp. 813-820, May 1993.

[32] A. Yuan and B. S. Clarke, "An information criterion for likelihood selection,” IEEE Trans. Inform. Theory, vol. 45, pp. 562-571, Mar. 1999.

[33] R. Zamir and K. Rose, "A type generator model for adaptive lossy compression," in Proc. IEEE Int. Symp. Information Theory, Ulm, Germany, June/July 1997, p. 186

[34] — - "Natural type selection in adaptive lossy compression," IEEE Trans. Inform. Theory, vol. 47, pp. 99-111, Jan. 2001.

[35] Z. Zhang, E.-h. Yang, and V. K. Wei, "The redundancy of source coding with a fidelity criterion-Part I: Known statistics," IEEE Trans. Inform. Theory, vol. 43, pp. 71-91, Jan. 1997.

[36] C. E. Shannon, "Coding theorems for a discrete source with a fidelity criterion," in Key Papers in the Development of Information Theory, D. Slepian, Ed. New York: IEEE Press, 1974. 